\title{
Genus-zero two-point hyperplane integrals in the Gromov-Witten theory
}

\author{
Aleksey Zinger
}

\begin{abstract}
In this paper, we compute certain two-point integrals over a moduli space of stable maps into projective space. Computation of onepoint analogs of these integrals constitutes a proof of mirror symmetry for genus-zero one-point Gromov-Witten (GW) invariants of projective hypersurfaces. The integrals computed in this paper constitute a significant portion in the proof of mirror symmetry for genus-one $\mathrm{GW}$-invariants completed in a separate paper. These integrals also provide explicit mirror formulas for genus-zero twopoint GW-invariants of projective hypersurfaces. The approach described in this paper leads to a reconstruction algorithm for all genus-zero GW-invariants of projective hypersurfaces.
\end{abstract}

1 Introduction $\quad 956$

$\begin{array}{lll}1.1 & \text { Background and motivation } & 956\end{array}$

$\begin{array}{lll}1.2 & \text { Main theorem } & 961\end{array}$

$\begin{array}{lll}\text { 1.3 Outline of the proof } & 964\end{array}$

2 Algebraic observations $\quad \mathbf{9 7 0}$

$\begin{array}{lll}2.1 & \text { On rigidity of certain polynomial conditions } & 970\end{array}$

$\begin{array}{lll}2.2 & \text { Admissible transforms } & 976\end{array}$

$\begin{array}{lll}2.3 & \text { Some properties of hypergeometric series } & 979\end{array}$

3 Localization computations $\quad 982$

$\begin{array}{lll}3.1 & \text { Equivariant cohomology } & 982\end{array}$ 


\section{Introduction}

\subsection{Background and motivation}

The theory of Gromov-Witten (GW) invariants has been greatly influenced by its interactions with string theory. In particular, the mirror symmetry principle has led to completely unexpected predictions concerning GWinvariants of Calabi-Yau manifolds. The original prediction of [4] for the genus-zero $\mathrm{GW}$-invariants of a quintic three-fold was verified about ten years ago in a variety of ways in $[3,5,6,8,10]$. The 1993 prediction of [2] for the genus-one GW-invariants of a quintic three-fold is verified in [14], using the results of this paper.

The proof of the genus-zero mirror symmetry for a projective hypersurface $X$ essentially consists of computing certain equivariant integrals on moduli spaces $\overline{\mathfrak{M}}_{0, m}\left(\mathbb{P}^{n-1}, d\right)$ of stable degree- $d$ maps from genus-zero curves with $m$ marked points into $\mathbb{P}^{n-1}$. While the integrals appearing in Chapters 29 and 30 of $[11]$ are over $\overline{\mathfrak{M}}_{0,2}\left(\mathbb{P}^{n-1}, d\right)$, the integrands involve only one marked point. For this reason, such integrals can be easily expressed in terms of integrals on $\overline{\mathfrak{M}}_{0,1}\left(\mathbb{P}^{n-1}, d\right)$ and determine genus-zero one-point GW-invariants of $X$; see (1.2) below. In this paper, we compute integrals on $\overline{\mathfrak{M}}_{0,2}\left(\mathbb{P}^{n-1}, d\right)$ with integrands involving both marked points. These integrals in a sense correspond to arithmetic genus one and indeed constitute a significant portion of the proof of mirror symmetry for genus-one GW-invariants in [14]. Theorem 1.1 also provides closed mirror formulas for genus-zero two-point $\mathrm{GW}$-invariants of $X$, including with descendants. At the end of Section 1.3, we describe the issue arising for integrals with more marked points, a potential way of addressing it, and a reconstruction algorithm for genus-zero GW-invariants of $X$ with descendants. 
Let $\mathfrak{U}$ be the universal curve over $\overline{\mathfrak{M}}_{0, m}\left(\mathbb{P}^{n-1}, d\right)$, with structure map $\pi$ and evaluation map ev:

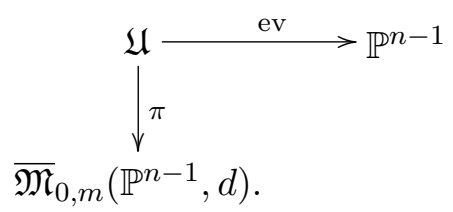

In other words, the fiber of $\pi$ over $[\mathcal{C}, f]$ is the curve $\mathcal{C}$ with $m$ marked points, while

$$
\mathrm{ev}([\mathcal{C}, f ; z])=f(z) \quad \text { if } \quad z \in \mathcal{C}
$$

If $a$ is a positive integer, the sheaf

$$
\pi_{*} \operatorname{ev}^{*} \mathcal{O}_{\mathbb{P}^{n-1}}(a) \longrightarrow \overline{\mathfrak{M}}_{0, m}\left(\mathbb{P}^{n-1}, d\right)
$$

is locally free. We denote the corresponding vector bundle by $\mathcal{V}_{0} \cdot{ }^{1}$ Its Euler class, $e\left(\mathcal{V}_{0}\right)$, relates genus-zero GW-invariants of a degree- $a$ hypersurface in $\mathbb{P}^{n-1}$ to genus-zero GW-invariants of $\mathbb{P}^{n-1}$; see Section 26.1 in [11]. For each $i=1, \ldots, m$, there is a well-defined bundle map

$$
\widetilde{\mathrm{ev}}_{i}: \mathcal{V}_{0} \longrightarrow \operatorname{ev}_{i}^{*} \mathcal{O}_{\mathbb{P}^{n-1}}(a), \quad \widetilde{\mathrm{ev}}_{i}([\mathcal{C}, f ; \xi])=\left[\xi\left(x_{i}(\mathcal{C})\right)\right]
$$

where $x_{i}(\mathcal{C})$ is the $i$ th marked of $\mathcal{C}$. Since it is surjective, its kernel is again a vector bundle. Let

$$
\mathcal{V}_{0}^{\prime}=\operatorname{ker} \tilde{\mathrm{ev}}_{1} \longrightarrow \overline{\mathfrak{M}}_{0, m}\left(\mathbb{P}^{n-1}, d\right) \quad \text { and } \quad \mathcal{V}_{0}^{\prime \prime}=\operatorname{ker} \tilde{\mathrm{ev}}_{2} \longrightarrow \overline{\mathfrak{M}}_{0, m}\left(\mathbb{P}^{n-1}, d\right)
$$

whenever $m \geq 1$ and $m \geq 2$, respectively. ${ }^{2}$

The standard action of the $n$-torus $\mathbb{T}$ on $\mathbb{P}^{n-1}$ induces $\mathbb{T}$-actions on $\overline{\mathfrak{M}}_{0, m}\left(\mathbb{P}^{n-1}, d\right), \mathfrak{U}, \mathcal{V}_{0}, \mathcal{V}_{0}^{\prime}$ and $\mathcal{V}_{0}^{\prime \prime}$; see Sections 3.1 and 3.2 for details on

${ }^{1}$ The fiber of $\mathcal{V}_{0}$ over a point $[\mathcal{C}, f] \in \overline{\mathfrak{M}}_{0, m}\left(\mathbb{P}^{n-1}, d\right)$ is $H^{0}\left(\mathcal{C} ; f^{*} \mathcal{O}_{\mathbb{P}^{n-1}}(a)\right) /$ $\operatorname{Aut}(\mathcal{C}, f)$.

${ }^{2}$ In Chapters 29 and 30 of [11], the roles of the marked points 1 and 2 in (1.2) are switched; the analogs of $\mathcal{V}_{0}$ and $\mathcal{V}_{0}^{\prime}$ over $\overline{\mathfrak{M}}_{0,2}\left(\mathbb{P}^{n}, d\right)$ are denoted by $E_{0, d}$ and $E_{0, d}^{\prime}$, respectively. 
equivariant cohomology. In particular, $\mathcal{V}_{0}, \mathcal{V}_{0}^{\prime}$ and $\mathcal{V}_{0}^{\prime \prime}$ have well-defined equivariant Euler classes

$$
\mathbf{e}\left(\mathcal{V}_{0}\right), \mathbf{e}\left(\mathcal{V}_{0}^{\prime}\right), \mathbf{e}\left(\mathcal{V}_{0}^{\prime \prime}\right) \in H_{\mathbb{T}}^{*}\left(\overline{\mathfrak{M}}_{0, m}\left(\mathbb{P}^{n-1}, d\right)\right)
$$

These classes are related by

$$
\mathbf{e}\left(\mathcal{V}_{0}\right)=a \operatorname{ev}_{1}^{*}(x) \mathbf{e}\left(\mathcal{V}_{0}^{\prime}\right)=a \mathrm{ev}_{2}^{*}(x) \mathbf{e}\left(\mathcal{V}_{0}^{\prime \prime}\right)
$$

where $x \in H_{\mathbb{T}}^{*}\left(\mathbb{P}^{n-1}\right)$ is the equivariant hyperplane class. For each $i=1,2$, $\ldots, m$, there is also a well-defined equivariant $\psi$-class,

$$
\psi_{i} \in H_{\mathbb{T}}^{2}\left(\overline{\mathfrak{M}}_{0, m}\left(\mathbb{P}^{n-1}, d\right)\right),
$$

the first chern of the vertical cotangent line bundle of $\mathfrak{U}$ pull-backed to $\overline{\mathfrak{M}}_{0, m}\left(\mathbb{P}^{n-1}, d\right)$ by the section

$$
\overline{\mathfrak{M}}_{0, m}\left(\mathbb{P}^{n-1}, d\right) \longrightarrow \mathfrak{U}, \quad[\mathcal{C}, f] \longrightarrow\left[\mathcal{C}, f ; x_{i}(\mathcal{C})\right]
$$

Since $\overline{\mathfrak{M}}_{0, m}\left(\mathbb{P}^{n-1}, d\right)$ is a smooth stack (orbifold), there is an integrationalong-the-fiber homomorphism

$$
\int_{\overline{\mathfrak{M}}_{0, m}\left(\mathbb{P}^{n-1}, d\right)}: H_{\mathbb{T}}^{*}\left(\overline{\mathfrak{M}}_{0, m}\left(\mathbb{P}^{n-1}, d\right)\right) \longrightarrow H_{\mathbb{T}}^{*} \approx \mathbb{Q}\left[\alpha_{1}, \ldots, \alpha_{n}\right] .
$$

For each $i=1,2, \ldots, n$, let

$$
\phi_{i} \in H_{\mathbb{T}}^{*}\left(\mathbb{P}^{n-1}\right) \approx \mathbb{Q}\left[x, \alpha_{1}, \ldots, \alpha_{n}\right] /\left(x-\alpha_{1}\right) \ldots\left(x-\alpha_{n}\right)
$$

be the equivariant Poincare dual of the $i$ th fixed point $P_{i} \in \mathbb{P}^{n-1}$. Let

$$
\mathbb{Q}_{\alpha} \equiv \mathbb{Q}\left(\alpha_{1}, \ldots, \alpha_{n}\right)
$$

denote the field of fractions in $\alpha_{1}, \ldots, \alpha_{n}$. For $a=1,2, \ldots, n$, an explicit algebraic formula for

$$
\begin{aligned}
\mathcal{Z}\left(\hbar, \alpha_{i}, u\right) & \equiv 1+\sum_{d=1}^{\infty} u^{d} \int_{\overline{\mathfrak{M}}_{0,2}\left(\mathbb{P}^{n-1}, d\right)} \frac{\mathbf{e}\left(\mathcal{V}_{0}^{\prime}\right)}{\hbar-\psi_{1}} \mathrm{ev}_{1}^{*} \phi_{i} \\
& =\hbar^{-1}\left(\hbar+\sum_{d=1}^{\infty} u^{d} \int_{\overline{\mathfrak{M}}_{0,1}\left(\mathbb{P}^{n-1}, d\right)} \frac{\mathbf{e}\left(\mathcal{V}_{0}^{\prime}\right)}{\hbar-\psi_{1}} \operatorname{ev}_{1}^{*} \phi_{i}\right) \in\left(\mathbb{Q}_{\alpha}\left[\left[\hbar^{-1}\right]\right]\right)[[u]]
\end{aligned}
$$


is confirmed in Chapters 29 and 30 of [11]. The equality in (1.2) is a straightforward consequence of the string relation for GW-invariants; see Section 26.3 in [11].

One of the ingredients in genus-one localization computations is a twopointed version of (1.2):

$$
\begin{aligned}
\widetilde{\mathcal{Z}}\left(\hbar_{1}, \hbar_{2}, \alpha_{i}, \alpha_{j}, u\right) \equiv & \frac{a \alpha_{i}}{\hbar_{1}+\hbar_{2}} \prod_{k \neq i}\left(\alpha_{j}-\alpha_{k}\right) \\
& +\sum_{d=1}^{\infty} u^{d} \int_{\overline{\mathfrak{M}}_{0,2}\left(\mathbb{P}^{n-1}, d\right)} \frac{\mathbf{e}\left(\mathcal{V}_{0}\right) \mathrm{ev}_{1}^{*} \phi_{i} \mathrm{ev}_{2}^{*} \phi_{j}}{\left(\hbar_{1}-\psi_{1}\right)\left(\hbar_{2}-\psi_{2}\right)} \\
& \in\left(\mathbb{Q}_{\alpha}\left[\left[\hbar_{1}^{-1}, \hbar_{2}^{-1}\right]\right]\right)[[u]]
\end{aligned}
$$

Note that the term of degree zero in $u$ above is symmetric in $\left(\hbar_{1}, \alpha_{i}\right)$ and $\left(\hbar_{2}, \alpha_{j}\right)$, just as are the positive-degree terms. In turn, $\widetilde{\mathcal{Z}}\left(\hbar_{1}, \hbar_{2}, \alpha_{i}, \alpha_{j}, u\right)$ can be determined from the power series

$$
\begin{aligned}
\mathcal{Z}_{p}\left(\hbar, \alpha_{i}, u\right) \equiv & \alpha_{i}^{p+1}+\sum_{d=1}^{\infty} u^{d} \int_{\bar{M}_{0,2}\left(\mathbb{P}^{n-1}, d\right)} \frac{\mathbf{e}\left(\mathcal{V}_{0}^{\prime \prime}\right) \mathrm{ev}_{2}^{*} x^{p+1}}{\hbar-\psi_{1}} \operatorname{ev}_{1}^{*} \phi_{i} \\
& \in\left(\mathbb{Q}_{\alpha}\left[\left[\hbar^{-1}\right]\right]\right)[[u]]
\end{aligned}
$$

with $p=-1,0, \ldots, n-1$. The seemingly unfortunate choice of indexing is partly motivated by the central role played by the power series $\mathcal{Z}\left(\hbar, \alpha_{i}, u\right)$ defined in (1.2) and the simple relation

$$
\mathcal{Z}_{0}\left(\hbar, \alpha_{i}, u\right)=\alpha_{i} \mathcal{Z}\left(\hbar, \alpha_{i}, u\right),
$$

which follows from (1.1), along with (3.9), (3.4) and (3.5). As shown in this paper,

$$
\widetilde{\mathcal{Z}}\left(\hbar_{1}, \hbar_{2}, \alpha_{i}, \alpha_{j}, u\right)=\frac{a}{\hbar_{1}+\hbar_{2}} \sum_{p+q+r=n-1}(-1)^{r} \sigma_{r} \mathcal{Z}_{p}\left(\hbar_{1}, \alpha_{i}, u\right) \mathcal{Z}_{q-1}\left(\hbar_{2}, \alpha_{j}, u\right)
$$

where $\sigma_{p}$ is the $p$ th elementary symmetric polynomial in $\alpha_{1}, \ldots, \alpha_{n}$; see Theorem 1.1.

Remark 1. The right-hand side of (1.5) is in fact symmetric in $\left(\hbar_{1}, \alpha_{i}\right)$ and $\left(\hbar_{2}, \alpha_{j}\right)$, because

$$
\mathcal{Z}_{n-1}\left(\hbar, \alpha_{i}, u\right)-\sigma_{1} \mathcal{Z}_{n-2}\left(\hbar, \alpha_{i}, u\right)+\ldots+(-1)^{n} \sigma_{n} \mathcal{Z}_{-1}\left(\hbar, \alpha_{i}, u\right)=0 .
$$

The reason for this relation is explained in Section 1.3. 
Table 1: Some genus-zero two-point BPS numbers.

\begin{tabular}{|c|c|}
\hline $\begin{array}{l}\text { Degree } \\
d\end{array}$ & $\begin{array}{l}\text { BPS curve count through } 2 \text { codim-2 } \\
\text { linear subspaces in } X_{7}\end{array}$ \\
\hline 1 & 1707797 \\
\hline 2 & 510787745643 \\
\hline 3 & 222548537108926490 \\
\hline 4 & 113635631482486991647224 \\
\hline 5 & 63340724462384110502639024265 \\
\hline 6 & 37325795060717360046547665187418254 \\
\hline 7 & 22857028298936684292245509537579343818647 \\
\hline 8 & 14395953469762596243721601709186933042635134584 \\
\hline 9 & 9263611884884554518268724722981763557936573405648178 \\
\hline 10 & 6062677702410680024315392235188823274104219383883410807999 \\
\hline
\end{tabular}

Remark 2. We will see in Section 1.2 that the power series $\mathcal{Z}_{p}\left(\hbar, \alpha_{i}, u\right)$ can be represented by elements of $\mathbb{Q}_{\alpha}(\hbar)[[u]]$. Relation (1.5) might then suggest that the corresponding element of $\mathbb{Q}_{\alpha}\left(\hbar_{1}, \hbar_{2}\right)[[u]]$ representing $\widetilde{\mathcal{Z}}\left(\hbar_{1}, \hbar_{2}\right.$, $\left.\alpha_{i}, \alpha_{j}, u\right)$ has a simple pole at $\hbar_{1}=-\hbar_{2}$. In fact, there is no pole at $\hbar_{1}=-\hbar_{2}$, except in degree zero. This is immediate from the localization formula (3.9); see also Section 3.2.

The power series (1.4) encode genus-zero two-point GW-invariants of a degree- $a$ hypersurface in $\mathbb{P}^{n-1}$ with constraints coming from $\mathbb{P}^{n-1}$. Thus, Theorem 1.1 provides mirror formulas for such invariants; the coefficients $\tilde{C}_{p, q}^{(r)}$ are "purely equivariant" and are irrelevant for this purpose. In Table 1, we give the first ten genus-zero two-point BPS numbers, defined from GW-invariants by Equation (2) in [7], for the degree-7 hypersurface in $\mathbb{P}^{6}$. These numbers are integers as predicted by Conjecture 1 in [7]. In fact, we have used the first statement of Theorem 1.1, along with a computer program, to confirm this conjecture for all degree- $d$ two-point BPS counts in a degree- $n$ hypersurface $X_{n}$ in $\mathbb{P}^{n-1}$ with $n \leq 10$ and $d \leq 20$.

The explicit expressions of Section 29.1 in [11] for the power series $\mathcal{Z}\left(\hbar, \alpha_{i}, u\right)$ have very different forms for $a<n$ and $a=n$. The $a=n$ case is the most interesting one and corresponds to Calabi-Yau hypersurfaces. As the power series $\mathcal{Z}\left(\hbar, \alpha_{i}, u\right)$ are central to our computation of $\mathcal{Z}_{p}(\hbar, \alpha, u)$ and $\widetilde{\mathcal{Z}}\left(\hbar_{1}, \hbar_{2}, \alpha_{i}, \alpha_{j}, u\right)$, for the purposes of the explicit expressions preceding 
Theorem 1.1 in the next subsection we consider only the case $a=n .{ }^{3}$ This is also the case used in [14].

\subsection{Main theorem}

The essence of mirror symmetric predictions for GW invariants is that these invariants (and relatedly $\mathcal{Z}\left(\hbar, \alpha_{i}, u\right)$ ) can be expressed in terms of certain hypergeometric series. In this subsection, we define these series and then express $\mathcal{Z}\left(\hbar, \alpha_{i}, u\right), \mathcal{Z}_{p}\left(\hbar, \alpha_{i}, u\right)$ and $\widetilde{\mathcal{Z}}\left(\hbar_{1}, \hbar_{2}, \alpha_{i}, \alpha_{j}, u\right)$ in terms of them.

Let $n$ be a positive integer. For each $q=0,1, \ldots$, define $I_{0, q}(t)$ by

$$
\sum_{q=0}^{\infty} I_{0, q}(t) w^{q} \equiv e^{w t} \sum_{d=0}^{\infty} e^{d t} \frac{\prod_{r=1}^{r=n d}(n w+r)}{\prod_{r=1}^{r=d}(w+r)^{n}}
$$

Each $I_{0, q}(t)$ is a degree- $q$ polynomial in $t$ with coefficients that are power series in $e^{t}$. For example,

$$
I_{0}(t)=1+\sum_{d=1}^{\infty} e^{d t} \frac{(n d) !}{(d !)^{n}}, \quad I_{1}(t)=t I_{0}(t)+\sum_{d=1}^{\infty} e^{d t}\left(\frac{(n d) !}{(d !)^{n}} \sum_{r=d+1}^{n d} \frac{n}{r}\right)
$$

For $p, q \in \mathbb{Z}^{+}$with $q \geq p$, let

$$
I_{p, q}(t)=\frac{d}{d t}\left(\frac{I_{p-1, q}(t)}{I_{p-1, p-1}(t)}\right) .
$$

It is straightforward to check that each of the "diagonal" terms $I_{p, p}(t)$ is a power series in $e^{t}$ with constant term 1 , whenever it is defined; see [13], for example. Thus, the division in (1.9) is well-defined for all $p$. Let

$$
T=\frac{I_{0,1}(t)}{I_{0,0}(t)}
$$

By (1.8), the map $t \longrightarrow T$ is a change of variables; it will be called the mirror map. If $p \in \overline{\mathbb{Z}}^{+}$and $\mathcal{Y}\left(\hbar, x, e^{t}\right)$ is a power series in $e^{t}$ with coefficients

\footnotetext{
${ }^{3}$ In other words, one may to choose set $a=n$ for the rest of the paper. However, the statements of Lemmas 1.1 and 1.2 and their proofs are valid for all $a$. Therefore, the proofs of (1.5) and (1.6) are valid as well. The same is the case with Theorem 1.1 if the power series $\mathcal{Y}_{-1}$ and $\mathcal{Y}$ are chosen appropriately.
} 
that are functions of a complex variable $\hbar$ and possibly some other variable $x$, let

$$
\mathfrak{D}^{p} \mathcal{Y}(\hbar, x, t)=e^{-x t / \hbar}\left\{\frac{\hbar}{I_{p, p}(t)} \frac{d}{d t}\right\} \cdots\left\{\frac{\hbar}{I_{1,1}(t)} \frac{d}{d t}\right\}\left(e^{x t / \hbar} \mathcal{Y}\left(\hbar, x, e^{t}\right)\right)
$$

We define

$$
\begin{aligned}
\mathcal{Y}\left(\hbar, x, e^{t}\right)= & I_{0,0}(t)^{-1} x \sum_{d=0}^{\infty} e^{d t} \frac{\prod_{r=1}^{r=n d}(n x+r \hbar)}{\prod_{r=1}^{r=d} \prod_{k=1}^{k=n}\left(x-\alpha_{k}+r \hbar\right)} \\
& \in \mathbb{Q}_{\alpha}(\hbar, x)\left[\left[e^{t}\right]\right] / \prod_{k=1}^{k=n}\left(x-\alpha_{k}\right) .
\end{aligned}
$$

Expanding $e^{x t / \hbar} \mathcal{Y}\left(\hbar, x, e^{t}\right)$ as a power series in $\hbar^{-1}$, we obtain

$$
e^{x t / \hbar} \mathcal{Y}\left(\hbar, x, e^{t}\right)=x \sum_{q=0}^{\infty}\left(\sum_{r=0}^{r=q} C_{0, q}^{(r)}(t) x^{q-r}\right) \hbar^{-q}
$$

where $C_{0, q}^{(r)}(t)$ is a degree- $r$ symmetric polynomial in $\alpha_{1}, \ldots, \alpha_{n}$ with coefficients in $\mathbb{Q}[t]\left[\left[e^{t}\right]\right]$. For example,

$$
C_{0, q}^{(0)}(t)=I_{0, q}(t) / I_{0,0}(t), \quad C_{0,1}^{(1)}(t)=\sigma_{1} I_{0,0}(t)^{-1} \sum_{d=1}^{\infty} e^{d t}\left(\frac{(n d) !}{(d !)^{n}} \sum_{r=1}^{n} \frac{1}{r}\right)
$$

The main conclusion of Section 30.4 in [11] is that the power series $\mathcal{Z}\left(\hbar, \alpha_{i}, e^{T}\right)$ defined in (1.2) is the evaluation of

$$
\mathcal{Z}\left(\hbar, x, e^{T}\right)=e^{(t-T) x / \hbar} e^{-C_{0,1}^{(1)}(t) / \hbar} \mathcal{Y}\left(\hbar, x, e^{t}\right) \in \mathbb{Q}_{\alpha}(\hbar, x)\left[\left[e^{t}\right]\right] / \prod_{k=1}^{k=n}\left(x-\alpha_{k}\right)
$$

at $x=\alpha_{i}$, if $T$ and $t$ are related by the mirror map (1.10).

The power series $\mathcal{Z}_{p}\left(\hbar, \alpha_{i}, u\right)$ and $\widetilde{\mathcal{Z}}\left(\hbar_{1}, \hbar_{2}, \alpha_{i}, \alpha_{j}, u\right)$ defined in (1.4) and (1.3), respectively, are also evaluations of certain power series

$$
\mathcal{Z}_{p}(\hbar, x, u) \in \mathbb{Q}_{\alpha}(\hbar, x)[[u]] / \prod_{k=1}^{k=n}\left(x-\alpha_{k}\right)
$$


and

$$
\widetilde{\mathcal{Z}}\left(\hbar_{1}, \hbar_{2}, x_{1}, x_{2}, u\right) \in \mathbb{Q}_{\alpha}\left(\hbar_{1}, \hbar_{2}, x_{1}, x_{2}\right)[[u]] / \prod_{k=1}^{k=n}\left(x_{1}-\alpha_{k}\right)\left(x_{2}-\alpha_{k}\right)
$$

that have a mirror transform shape analogous to (1.14). Let

$$
\begin{aligned}
\mathfrak{D}^{-1} \mathcal{Y}\left(\hbar, x, e^{t}\right) \equiv & \sum_{d=0}^{\infty} e^{d t} \frac{\prod_{r=0}^{n d-1}(n x+r \hbar)}{\prod_{r=1}^{r=d} \prod_{k=1}^{k=n}\left(x-\alpha_{k}+r \hbar\right)} \\
& \in \mathbb{Q}_{\alpha}(\hbar, x)\left[\left[e^{t}\right]\right] / \prod_{k=1}^{k=n}\left(x-\alpha_{k}\right) .
\end{aligned}
$$

Theorem 1.1. There exist $\tilde{C}_{p, q}^{(r)} \in \mathbb{Q}_{\alpha}\left[\left[e^{t}\right]\right]$, with $p \geq r \geq 1$ and $p-r \geq q \geq$ 0 , such that the coefficients of the powers of $e^{t}$ in $\tilde{C}_{p, q}^{(r)}$ are degree-r symmetric polynomials and the power series defined in (1.4) are given by

$$
\mathcal{Z}_{p}\left(\hbar, x, e^{T}\right)=e^{(t-T) x / \hbar} e^{-C_{0,1}^{(1)}(t) / \hbar} \mathcal{Y}_{p}\left(\hbar, x, e^{t}\right)
$$

where

$$
\mathcal{Y}_{p}\left(\hbar, x, e^{t}\right) \equiv \mathfrak{D}^{p} \mathcal{Y}\left(\hbar, x, e^{t}\right)+\sum_{r=1}^{r=p} \sum_{q=0}^{p-r} \tilde{C}_{p, q}^{(r)}\left(e^{t}\right) \hbar^{p-r-q} \mathfrak{D}^{q} \mathcal{Y}\left(\hbar, x, e^{t}\right),
$$

if $T$ and $t$ are related by the mirror map (1.10). Furthermore, the power series defined in (1.3) are given by

$$
\widetilde{\mathcal{Z}}\left(\hbar_{1}, \hbar_{2}, x_{1}, x_{2}, u\right)=\frac{a}{\hbar_{1}+\hbar_{2}} \sum_{p+q+r=n-1}(-1)^{r} \sigma_{r} \mathcal{Z}_{p}\left(\hbar_{1}, x_{1}, u\right) \mathcal{Z}_{q-1}\left(\hbar_{2}, x_{2}, u\right)
$$

Remark. We note that by (1.16) and (1.17)

$\widetilde{\mathcal{Z}}\left(\hbar_{1}, \hbar_{2}, x_{1}, x_{2}, e^{T}\right)=e^{(t-T)\left(x_{1} / \hbar_{1}+x_{2} / \hbar_{2}\right)} e^{-C_{0,1}^{(1)}(t)\left(\hbar_{1}^{-1}+\hbar_{2}^{-1}\right)} \widetilde{\mathcal{Y}}\left(\hbar_{1}, \hbar_{2}, x_{1}, x_{2}, e^{t}\right)$

where

$$
\widetilde{\mathcal{Y}}\left(\hbar_{1}, \hbar_{2}, x_{1}, x_{2}, u\right) \equiv \frac{a}{\hbar_{1}+\hbar_{2}} \sum_{p+q+r=n-1}(-1)^{r} \sigma_{r} \mathcal{Y}_{p}\left(\hbar_{1}, x_{1}, u\right) \mathcal{Y}_{q-1}\left(\hbar_{2}, x_{2}, u\right)
$$

In other words, $\widetilde{\mathcal{Z}}$ is the same transform of $\widetilde{\mathcal{Y}}$ in both $\left(x_{1}, \hbar_{1}\right)$ and $\left(\hbar_{2}, x_{2}\right)$ as $\mathcal{Z}$ and $\mathcal{Z}_{p}$ are of $\mathcal{Y}$ and $\mathcal{Y}_{p}$ in $(\hbar, x)$. 
The only relevant property of the power series $\tilde{C}_{p, q}^{(r)}$ for the purposes of the genus-one localization computations in [14] is that the $e^{t}$-coefficients of $\tilde{C}_{p, q}^{(r)}$ lie in the ideal generated by $\sigma_{1}, \ldots, \sigma_{n-1}$ if $p \leq n-1$. This is automatic in the case of Theorem 1.1, since each of these coefficients is a symmetric polynomial in $\alpha_{1}, \ldots, \alpha_{n}$ of a positive degree $r \leq n-1$. The author's approach in [14] suggests that these coefficients are likely to be irrelevant in many other localization computations as well. Nevertheless, they are described inductively in this paper in the process of proving the first statement of Theorem 1.1.

\subsection{Outline of the proof}

The proof of (1.14) in Chapter 30 of [11] essentially consists of showing that

(S1) $\mathcal{Y}(\hbar, x, u)$ and $\mathcal{Z}(\hbar, x, u)$ satisfy a certain recursion on the $u$-degree;

$(\mathrm{S} 2) \mathcal{Y}(\hbar, x, u)$ and $\mathcal{Z}(\hbar, x, u)$ satisfy a certain self-polynomiality condition (SPC);

(S3) the two sides of (1.14), viewed as power series in $\hbar^{-1}$, agree $\bmod \hbar^{-2}$;

(S4) if $Y(\hbar, x, u)$ satisfies the recursion and the SPC, so do certain transforms of $Y(\hbar, x, u)$;

(S5) if $Y(\hbar, x, u)$ satisfies the recursion and the SPC, it is determined by its "mod $\hbar^{-2}$ part".

For the purposes of these statements, in particular (S3) and (S5), we assume that

$$
\mathcal{Y}(\hbar, x, u), \mathcal{Z}(\hbar, x, u), Y(\hbar, x, u) \in \mathbb{Q}_{\alpha}(\hbar, x)[[u]] / \prod_{k=1}^{k=n}\left(x-\alpha_{k}\right) .
$$

For example, (S5) means

$$
\begin{gathered}
Y\left(\hbar, \alpha_{i}, u\right) \cong \bar{Y}\left(\hbar, \alpha_{i}, u\right)\left(\bmod \hbar^{-2}\right) \quad \forall i=1,2, \ldots, n \\
\Longrightarrow Y\left(\hbar, \alpha_{i}, u\right)=\bar{Y}\left(\hbar, \alpha_{i}, u\right) \quad \forall i=1,2, \ldots, n .
\end{gathered}
$$

The proof of (1.16) in this paper essentially consists of showing that

(M1) $\mathcal{Y}_{p}(\hbar, x, u)$ and $\mathcal{Z}_{p}(\hbar, x, u)$ satisfy recursion $(2.13)$;

(M2) $\left(\mathcal{Y}(\hbar, x, u), \mathcal{Y}_{p}(\hbar, x, u)\right)$ and $\left(\mathcal{Z}(\hbar, x, u), \mathcal{Z}_{p}(\hbar, x, u)\right)$ satisfy the mutual polynomiality condition (MPC) of Lemma 2.2 ; 
(M3) the two sides of (1.16), viewed as power series in $\hbar^{-1}$, agree $\bmod \hbar^{-1}$;

(M4) if $Z(\hbar, x, u)$ satisfies (2.13) and the MPC with respect to $Y(\hbar, x, u)$, the transforms of $Z(\hbar, x, u)$ of Lemma 2.3 satisfy $(2.13)$ and the MPC with respect to appropriate transforms of $Y(\hbar, x, u)$;

(M5) if the $u$-constant term of $Y(\hbar, x, u)$ is independent of $\hbar$ and nonzero and $Z(\hbar, x, u)$ satisfies $(2.13)$ and the MPC with respect to $Y(\hbar, x, u)$, it is determined by its "mod $\hbar^{-1}$ part"; see Proposition 2.1.

Statements (M3) and (M5) should be interpreted analogously to (S3) and (S5). In other words, the equalities are modulo $\prod_{k=1}^{k=n}\left(x-\alpha_{k}\right)$, or equivalently after the evaluation at each $x=\alpha_{i}$. Similarly, the requirement on the degree-zero term in $Y(\hbar, x, u)$ in (M5) means that it is nonzero even after the evaluation at each $x=\alpha_{i}$.

The claims of (M1) and (M2) concerning $\mathcal{Z}_{p}(\hbar, x, u)$ are special cases of Lemmas 1.1 and 1.2 below, since

$$
\begin{aligned}
& \int_{\overline{\mathfrak{M}}_{0,2}\left(\mathbb{P}^{n-1}, d\right)} \frac{\mathbf{e}\left(\mathcal{V}_{0}^{\prime \prime}\right) \mathrm{ev}_{2}^{*} \eta}{\hbar-\psi_{1}} \mathrm{ev}_{1}^{*} \phi_{i} \\
& \quad=\hbar \int_{\overline{\mathfrak{M}}_{0,3}\left(\mathbb{P}^{n-1}, d\right)} \frac{\mathbf{e}\left(\mathcal{V}_{0}^{\prime \prime}\right) \mathrm{ev}_{2}^{*} \eta}{\hbar-\psi_{1}} \operatorname{ev}_{1}^{*} \phi_{i} \quad \forall \eta \in H_{\mathbb{T}}^{*}\left(\mathbb{P}^{n-1}\right), d \in \mathbb{Z}^{+}
\end{aligned}
$$

by the string relation; see Section 26.3 in [11].

Lemma 1.1. For all $m \geq 3, \eta_{j} \in H_{\mathbb{T}}^{*}\left(\mathbb{P}^{n-1}\right)$ and $\beta_{j} \in \overline{\mathbb{Z}}^{+}$, the power series $\mathcal{Z}_{\eta, \beta}(\hbar, x, u)$ defined by

$$
\mathcal{Z}_{\eta, \beta}(\hbar, x, u) \equiv \sum_{d=0}^{\infty} u^{d}\left(\int_{\overline{\mathfrak{M}}_{0, m}\left(\mathbb{P}^{n-1}, d\right)} \frac{\mathbf{e}\left(\mathcal{V}_{0}^{\prime \prime}\right) \mathrm{ev}_{1}^{*} \phi_{i}}{\hbar-\psi_{1}} \prod_{j=2}^{j=m}\left(\psi_{j}^{\beta_{j}} \mathrm{ev}_{j}^{*} \eta_{j}\right)\right)
$$

satisfies the recursion (2.13).

Lemma 1.2. For all $m \geq 3, \eta_{j} \in H_{\mathbb{T}}^{*}\left(\mathbb{P}^{n-1}\right)$ and $\beta_{j} \in \overline{\mathbb{Z}}^{+}$, the power series $\hbar^{m-2} \mathcal{Z}_{\eta, \beta}(\hbar, x, u)$, with $\mathcal{Z}_{\eta, \beta}(\hbar, x, u)$ as in Lemma 1.1 , satisfies the polynomial condition of Lemma 2.1 with respect to $\mathcal{Z}(\hbar, x, u)$.

Our proof of Lemma 1.1 is practically identical to the proof in Section 30.1 of $[11]$ that $\mathcal{Z}(\hbar, x, u)$ satisfies a certain recursion on the $u$-degree. ${ }^{4}$

\footnotetext{
${ }^{4}$ However, the coefficients $C_{i}^{j}(d)$ in $(2.13)$ are "shifts by one" of the coefficients in the recursion for $\mathcal{Z}(\hbar, x, u)$.
} 
The proof of Lemma 1.2 is similar to the proof in Section 30.2 of [11] that $\mathcal{Z}(\hbar, x, u)$ satisfies the SPC. However, there are differences in how the key idea for the setup used in [11] is utilized. An explanation of the modifications and a complete justification of their appropriateness are not very simple to state. In order to avoid any confusion, we thus give a full account in Sections 3.3 and 3.4. As it requires most of what constitutes the proof of the recursivity relation (2.13), we give a proof of the latter in Section 3.2.

We are now able to justify (1.6). By (1.4),

$$
\mathcal{Z}_{p}(\hbar, x, u) \cong x^{p+1} \quad\left(\bmod \hbar^{-1}\right) .^{5}
$$

Along with (M5), these three properties of $\mathcal{Z}_{p}(\hbar, x, u)$ imply (1.6), since

$$
\begin{aligned}
\sum_{p+r=n}(-1)^{r} \sigma_{r} \mathcal{Z}_{p-1}(\hbar, x, u) & \equiv \sum_{p+r=n}(-1)^{r} \sigma_{r} x^{p} \quad\left(\bmod \hbar^{-1}\right) \\
& =\prod_{k=1}^{k=n}\left(x-\alpha_{k}\right) .
\end{aligned}
$$

The last expression above vanishes at $x=\alpha_{i}$ for all $i=1,2, \ldots, n$.

We will check by a direct computation that

$$
\mathcal{Y}_{-1}(\hbar, x, u) \equiv \mathfrak{D}^{-1} \mathcal{Y}(\hbar, x, u)
$$

satisfies (2.13) and the MPC with respect to $\mathcal{Y}(\hbar, x, u)$; see Section 2.3. By (1.15),

$$
\mathcal{Y}_{-1}(\hbar, x, u) \cong 1 \quad\left(\bmod \hbar^{-1}\right) .
$$

Thus, the $p=-1$ case of (1.16) follows from (1.20), (M4) and (M5).

We could also verify directly that $\mathcal{Y}_{0}(\hbar, x, u)$ satisfies $(2.13)$ and the MPC with respect to $\mathcal{Y}(\hbar, x, u)$. Fortunately, this is an immediate consequence of parts (i) and (ii) of Lemma 2.3, since

$$
\mathcal{Y}_{0}\left(\hbar, x, e^{t}\right)=\frac{1}{I_{0,0}(t)}\left\{x+\hbar \frac{d}{d t}\right\} \mathcal{Y}_{-1}\left(\hbar, x, e^{t}\right)
$$

Thus, $\mathcal{Y}_{0}(\hbar, x, u)$ satisfies the two properties because $\mathcal{Y}_{-1}(\hbar, x, u)$ does. On the other hand, by (1.12) and (1.13),

$$
\mathcal{Y}_{0}(\hbar, x, u)=x \mathcal{Y}(\hbar, x, u) \cong x \quad\left(\bmod \hbar^{-1}\right) .
$$

\footnotetext{
${ }^{5}$ Such identities will be taken to mean that the two sides are equal if $x$ is replaced with $\alpha_{i}$ for every $i=1,2, \ldots, n$.
} 
Thus, the $p=0$ case of (1.16) also follows from (1.20), (M4) and (M5).

The differentiation transform (i) of Lemma 2.3 is the only one of the five "admissible" transforms that has no analog in Chapter 30 of [11]. ${ }^{6}$ The admissibility of this transform, along with that of (ii) of Lemma 2.3, implies that $\mathcal{Y}_{p}(\hbar, x, u)$ defined by the second equation in (1.16) satisfies recursion (2.13) and the MPC of Lemma 2.2 with respect to $\mathcal{Y}(\hbar, x, u)$, no matter what the coefficients $\tilde{C}_{p, q}^{(r)}(u)$ are. In light of $(1.20)$, the $p \geq 1$ cases of the first equation in (1.16) thus reduce to showing there exist $\tilde{C}_{p, q}^{(r)}(u)$ such that

$$
\mathcal{Y}_{p}(\hbar, x, u) \cong x^{p+1} \quad\left(\bmod \hbar^{-1}\right)
$$

This is proved by induction, using (1.12) and (1.13); see Section 2.3.

The proof of (1.17) follows the same principle. By the string relation and (1.1),

$$
\begin{aligned}
& \widetilde{\mathcal{Z}}\left(\hbar_{1}, \hbar_{2}, \alpha_{i}, \alpha_{j}, x_{2}, u\right) \\
& \quad=\frac{\hbar_{1} \hbar_{2}}{\hbar_{1}+\hbar_{2}} \sum_{d=0}^{\infty} \int_{\overline{\mathfrak{M}}_{0,3}\left(\mathbb{P}^{n-1}, d\right)} \frac{\mathbf{e}\left(\mathcal{V}_{0}^{\prime \prime}\right)}{\left(\hbar_{1}-\psi_{1}\right)\left(\hbar_{2}-\psi_{2}\right)} \mathrm{ev}_{1}^{*} \phi_{i} \mathrm{ev}_{2}^{*}\left(a x \phi_{j}\right) .
\end{aligned}
$$

Thus, by Lemmas 1.1 and $1.2,\left(\hbar_{1}+\hbar_{2}\right) \widetilde{\mathcal{Z}}\left(\hbar_{1}, \hbar_{2}, x_{1}, x_{2}, u\right)$ satisfies recursion (2.13) and the MPC of Lemma 2.2 with respect to $\mathcal{Z}(\hbar, x, u)$ for $(\hbar, x)=$ $\left(\hbar_{1}, x_{1}\right)$ and $x_{2}=\alpha_{j}$ fixed. By symmetry, it also satisfies the two properties for $(\hbar, x)=\left(\hbar_{2}, x_{2}\right)$ and $x_{1}=\alpha_{i}$ fixed. It is then sufficient to compare the two sides of (1.17) multiplied by $\hbar_{1}+\hbar_{2}$ modulo $\hbar_{1}^{-1}$ :

$$
\begin{aligned}
& \left(\hbar_{1}+\hbar_{2}\right) \widetilde{\mathcal{Z}}\left(\hbar_{1}, \hbar_{2}, \alpha_{i}, \alpha_{j}, u\right) \\
& \cong a \alpha_{i} \sum_{p+q+r=n-1}(-1)^{r} \sigma_{r} \alpha_{i}^{p} \alpha_{j}^{q}+\sum_{d=1}^{\infty} u^{d} \int_{\overline{\mathfrak{M}}_{0,2}\left(\mathbb{P}^{n-1}, d\right)} \frac{\mathbf{e}\left(\mathcal{V}_{0}\right) \mathrm{ev}_{1}^{*} \phi_{i} \mathrm{ev}_{2}^{*} \phi_{j}}{\hbar_{2}-\psi_{2}}, \\
& \quad \sum_{p+q+r=n-1}(-1)^{r} \sigma_{r} \mathcal{Z}_{p}\left(\hbar_{1}, \alpha_{i}, u\right) \mathcal{Z}_{q-1}\left(\hbar_{2}, \alpha_{j}, u\right) \\
& \quad a \sum_{p+q+r=n-1}(-1)^{r} \sigma_{r} \alpha_{i}^{p+1} \mathcal{Z}_{q-1}\left(\hbar_{2}, \alpha_{j}, u\right) .
\end{aligned}
$$

${ }^{6}$ The multiplication by $\hbar$, i.e., transform (iii), is not explicitly mentioned in Chapter 30 of [11], but its admissibility is nearly immediate from the relevant definitions. 
In order to see that the two resulting expressions are equal, we compare them modulo $\hbar_{2}^{-1}$ :

$$
\begin{aligned}
& a \sum_{p+q+r=n-1}(-1)^{r} \sigma_{r} \alpha_{i}^{p+1} \alpha_{j}^{q}+\sum_{d=1}^{\infty} u^{d} \int_{\overline{\mathfrak{M}}_{0,2}\left(\mathbb{P}^{n-1}, d\right)} \frac{\mathbf{e}\left(\mathcal{V}_{0}\right) \mathrm{ev}_{1}^{*} \phi_{i} \mathrm{ev}_{2}^{*} \phi_{j}}{\hbar_{2}-\psi_{2}} \\
& \quad \cong a \sum_{p+q+r=n-1}(-1)^{r} \sigma_{r} \alpha_{i}^{p+1} \alpha_{j}^{q}, \\
& a \sum_{p+q+r=n-1}(-1)^{r} \sigma_{r} \alpha_{i}^{p+1} \mathcal{Z}_{q-1}\left(\hbar_{2}, \alpha_{j}, u\right) \cong a \sum_{p+q+r=n-1}(-1)^{r} \sigma_{r} \alpha_{i}^{p+1} \alpha_{j}^{q} .
\end{aligned}
$$

From this we conclude that the two sides of (1.17) multiplied by $\hbar_{1}+\hbar_{2}$ modulo $\hbar_{1}^{-1}$ are equal. Therefore, the two sides (1.17) are equal by (M5).

Central to this paper are the use of the transforms $\mathfrak{D}^{p}$ in conjunction with part (i) of Lemma 2.3 and a desymmetrization of the approach of Chapter 30 of [11] to obtain an explicit closed formula for the integrals in (1.3). The transforms $\mathfrak{D}^{p}$, combined with transforms (ii) and (iii) of Lemma 2.3, make it possible to construct a power series $\widetilde{\mathcal{Y}}(\hbar, x, u)$, satisfying recursion $(2.13)$ and the MPC with respect to $\mathcal{Y}(\hbar, x, u)$, that agrees with a pre-specified $\alpha$-symmetric element of $\mathbb{Q}_{\alpha}[\hbar][[u]]$ modulo $\hbar^{-1}$. On the other hand, for the purposes of (M5), it is sufficient to assume that the coefficient of each power of $u$ in $Y$ and $Z$ is a sum of a power series in $\hbar^{-1}$ and a polynomial in $\hbar .^{7}$ Using (M5) and the last two parts of Lemma 2.3 , a variety of integrals on $\overline{\mathfrak{M}}_{0, m}\left(\mathbb{P}^{n}, d\right)$ involving $\mathbf{e}\left(\mathcal{V}_{0}\right)$ and products of $1 /\left(\hbar_{j}-\psi_{j}\right)$ can be reduced to integrals involving $\mathbf{e}\left(\mathcal{V}_{0}\right)$ and powers $\psi$-classes, with each exponent bounded by $m-3$.

For example, suppose one would like to compute

$$
\begin{aligned}
& \widetilde{\mathcal{Z}}^{(3)}\left(\hbar_{1}, \hbar_{2}, \hbar_{3}, \alpha_{i_{1}}, \alpha_{i_{2}}, \alpha_{i_{3}}, u\right) \\
& \equiv \sum_{d=0}^{\infty} u^{d} \int_{\overline{\mathfrak{M}}_{0,3}\left(\mathbb{P}^{n-1}, d\right)} \frac{\mathbf{e}\left(\mathcal{V}_{0}\right) \mathrm{ev}_{1}^{*} \phi_{i_{1}} \mathrm{ev}_{2}^{*} \phi_{i_{2}} \mathrm{ev}_{3}^{*} \phi_{i_{3}}}{\left(\hbar_{1}-\psi_{1}\right)\left(\hbar_{2}-\psi_{2}\right)\left(\hbar_{3}-\psi_{3}\right)},
\end{aligned}
$$

these integrals may be useful for localization computations in (arithmetic) genus two. By Lemmas 1.1 and 1.2 and part (iii) of Lemma $2.3, \hbar_{1} \hbar_{2} \hbar_{3} \widetilde{\mathcal{Z}}^{(3)}$ satisfies the recursion and the MPC with respect to $\mathcal{Z}$ for each $(\hbar, x)=$

\footnotetext{
${ }^{7}$ The same is the case with (S5). In fact, the condition on the middle term of the recursion used Chapter 30 of [11] can also be relaxed as in Definition 2.1.
} 
$\left(\hbar_{s}, x_{s}\right)$. Thus, $\widetilde{\mathcal{Z}}^{(3)}$ can be reconstructed ${ }^{8}$ from "mod $\hbar_{3}^{-1}$ " part of $\hbar_{1} \hbar_{2} \hbar_{3} \widetilde{\mathcal{Z}}^{(3)}$,

$$
\begin{aligned}
& \hbar_{1} \hbar_{2} \hbar_{3} \widetilde{\mathcal{Z}}^{(3)}\left(\hbar_{1}, \hbar_{2}, \hbar_{3}, \alpha_{i_{1}}, \alpha_{i_{2}}, \alpha_{i_{3}}, u\right) \\
& \cong \hbar_{1} \hbar_{2} \sum_{d=0}^{\infty} u^{d} \int_{\overline{\mathfrak{M}}_{0,3}\left(\mathbb{P}^{n-1}, d\right)} \frac{\mathbf{e}\left(\mathcal{V}_{0}\right) \mathrm{ev}_{1}^{*} \phi_{i_{1}} \mathrm{ev}_{2}^{*} \phi_{i_{2}} \mathrm{ev}_{3}^{*} \phi_{i_{3}}}{\left(\hbar_{1}-\psi_{1}\right)\left(\hbar_{2}-\psi_{2}\right)}
\end{aligned}
$$

once one computes

$$
\widetilde{\mathcal{Z}}_{p}\left(\hbar_{1}, \hbar_{2}, \alpha_{i_{1}}, \alpha_{i_{2}}, u\right) \equiv \sum_{d=0}^{\infty} u^{d} \int_{\overline{\mathfrak{M}}_{0,3}\left(\mathbb{P}^{n-1}, d\right)} \frac{\mathbf{e}\left(\mathcal{V}_{0}\right) \mathrm{ev}_{1}^{*} \phi_{i_{1}} \mathrm{ev}_{2}^{*} \phi_{i_{2}} \mathrm{ev}_{3}^{*} x^{p}}{\left(\hbar_{1}-\psi_{1}\right)\left(\hbar_{2}-\psi_{2}\right)}
$$

Similarly, $\hbar_{1} \hbar_{2} \widetilde{\mathcal{Z}}_{p}$ can be reconstructed from its "mod $\hbar_{2}^{-1}$ " part, if one can compute

$$
\mathcal{Z}_{p q}\left(\hbar_{1}, \alpha_{i_{1}}, u\right) \equiv \sum_{d=0}^{\infty} u^{d} \int_{\overline{\mathfrak{M}}_{0,3}\left(\mathbb{P}^{n-1}, d\right)} \frac{\mathbf{e}\left(\mathcal{V}_{0}\right) \mathrm{ev}_{1}^{*} \phi_{i_{1}} \mathrm{ev}_{2}^{*} x^{p} \mathrm{ev}_{3}^{*} x^{q}}{\hbar_{1}-\psi_{1}}
$$

Unfortunately, the "mod $\hbar_{1}^{-1}$ part" of $\mathcal{Z}_{p q}\left(\hbar_{1}, x, u\right)$ is not simple.

The above approach does, nevertheless, lead to a reconstruction theorem for $\mathbf{e}\left(\mathcal{V}_{0}\right)$-twisted GW-invariants of $\mathbb{P}^{n-1}$, or equivalently for $\mathrm{GW}$-invariants of projective hypersurfaces. The theorem arising here is different from $[9,12]$ for example, as the reduction is made to $\mathrm{GW}$-invariants with low powers of $\psi$-classes and without increasing the number of marked points. Furthermore, in may be possible to get a handle on the "components" of the "mod $\hbar_{1}^{-1}$ part" of $\mathcal{Z}_{p q}$, i.e.,

$$
\int_{\overline{\mathfrak{M}}_{0,3}\left(\mathbb{P}^{n-1}, d\right)} \mathrm{ev}_{1}^{*} x^{p} \mathrm{ev}_{2}^{*} x^{q} \mathrm{ev}_{3}^{*} x^{r},
$$

and $\mathcal{Z}_{p q}$ itself through the approach of Sections 2.4 and 2.5 in [14]. Along with (2.13), this approach leads to an explicit, but complicated, recursion for $\mathcal{Z}_{p q}$ (or the components of its "mod $\hbar_{1}^{-1}$ part").

$$
\begin{aligned}
& { }^{8} \text { From }(1.23) \text { and } \phi_{i_{3}}=\prod_{k \neq i_{3}}\left(x-\alpha_{i_{3}}\right) \text {, one obtains } \\
& \qquad \begin{array}{c}
\hbar_{1} \hbar_{2} \hbar_{3} \widetilde{\mathcal{Z}}^{(3)}\left(\hbar_{1}, \hbar_{2}, \hbar_{3}, x_{1}, x_{2}, x_{3}, u\right) \\
=\hbar_{1} \hbar_{2} \sum_{p+q+r=n-1}(-1)^{r} \sigma_{r} \widetilde{\mathcal{Z}}_{p}\left(\hbar_{1}, \hbar_{2}, x_{1}, x_{2}, u\right) \mathcal{Z}_{q}\left(\hbar_{3}, x_{3}, u\right)
\end{array}
\end{aligned}
$$




\section{Algebraic observations}

\subsection{On rigidity of certain polynomial conditions}

This subsection describes the extent of rigidity of power series with coefficients in rational functions that satisfy a certain recursion and a polynomiality condition. It is the analog of Section 30.3 in [11].

Denote by $\overline{\mathbb{Z}}^{+}$the set of nonnegative integers and by $[n]$, whenever $n \in$ $\overline{\mathbb{Z}}^{+}$, the set of positive number not exceeding $n$ :

$$
\overline{\mathbb{Z}}^{+}=\{0,1,2, \ldots,\}, \quad[n]=\{1,2, \ldots, n\} .
$$

Whenever $f$ is a function of $w$ (and possibly of other variables) which is holomorphic at $w=0$ (for a dense subspace of the other variables) and $s \in \overline{\mathbb{Z}}^{+}$, let

$$
\mathcal{D}_{w}^{s} f=\left.\frac{1}{s !}\left\{\frac{d}{d w}\right\}^{s} f(w)\right|_{w=0} .
$$

This is a function of the other variables if there are any.

Let $\tilde{\mathbb{Q}}_{\alpha}$ be any field extension of $\mathbb{Q}_{\alpha}$, possibly $\mathbb{Q}_{\alpha}$ itself. Given

$$
Y \equiv Y(\hbar, x, u), Z \equiv Z(\hbar, x, u) \in \tilde{\mathbb{Q}}_{\alpha}(\hbar, x)[[u]],
$$

we define

$$
\begin{aligned}
\Phi_{Y, Z} & \equiv \Phi_{Y, Z}(\hbar, u, z) \in \tilde{\mathbb{Q}}_{\alpha}(\hbar)[[u, z]] \text { by } \\
\Phi_{Y, Z}(\hbar, u, z) & =\sum_{i=1}^{i=n} \frac{e^{\alpha_{i} z}}{\prod_{k \neq i}\left(\alpha_{i}-\alpha_{k}\right)} Y\left(\hbar, \alpha_{i}, u e^{\hbar z}\right) Z\left(-\hbar, \alpha_{i}, u\right) .
\end{aligned}
$$

Lemma 2.1. If $Y, Z \in \tilde{\mathbb{Q}}_{\alpha}(\hbar, x)[[u]]$, there exists a unique collection

$$
\left(E_{Y, Z ; d} \equiv E_{Y, Z ; d}(\hbar, \Omega)\right)_{d \in \overline{\mathbb{Z}}^{+}} \subset \tilde{\mathbb{Q}}_{\alpha}(\hbar)[\Omega]
$$

such that the $\Omega$-degree of $E_{Y, Z ; d}$ is at most $(d+1) n-1$ for every $d \in \overline{\mathbb{Z}}^{+}$ and

$$
\Phi_{Y, Z}(\hbar, u, z)=\sum_{d=0}^{\infty} u^{d}\left(\frac{1}{2 \pi \mathfrak{i}} \oint e^{\Omega z} \frac{E_{Y, Z ; d}(\hbar, \Omega)}{\prod_{k=1}^{k=n} \prod_{r=0}^{r=d}\left(\Omega-\alpha_{k}-r \hbar\right)} d \Omega\right),
$$


where each path integral is taken over a simple closed loop in $\mathbb{C}$ enclosing all points $\Omega=\alpha_{k}+r \hbar$ with $k=1, \ldots, n$ and $r=0,1, \ldots, d$. The equality holds for a dense collection of complex parameters $\hbar$.

Proof. It can be assumed that

$$
\alpha_{k}+r \hbar \neq \alpha_{k^{\prime}}+r^{\prime} \hbar \quad \forall k, k^{\prime} \in[n], r, r^{\prime} \in \overline{\mathbb{Z}}^{+},(r, k) \neq\left(r^{\prime}, k^{\prime}\right) .
$$

Note that for every $i=1, \ldots, n$ and $d^{\prime}=0,1, \ldots, d$,

$$
\begin{aligned}
& \prod_{r=0}^{r=d^{\prime}-1}\left(\alpha_{i}+d^{\prime} \hbar-\alpha_{i}-r \hbar\right) \prod_{r=d^{\prime}+1}^{r=d}\left(\alpha_{i}+d^{\prime} \hbar-\alpha_{i}-r \hbar\right) \\
& \prod_{r=0}^{r=d} \prod_{k \neq i}\left(\alpha_{i}+d^{\prime} \hbar-\alpha_{k}-r \hbar\right) \\
& =d^{\prime} ! \hbar^{d^{\prime}}\left(d-d^{\prime}\right) !(-\hbar)^{d-d^{\prime}}\left(\prod_{r=1}^{r=d^{\prime}} \prod_{k \neq i}\left(\alpha_{i}-\alpha_{k}+r \hbar\right)\right)\left(\prod_{k \neq i}\left(\alpha_{i}-\alpha_{k}\right)\right) \\
& \quad\left(\prod_{r=1}^{r=d-d^{\prime}} \prod_{k \neq i}\left(\alpha_{i}-\alpha_{k}-r \hbar\right)\right) \\
& =\left(\prod_{k \neq i}\left(\alpha_{i}-\alpha_{k}\right)\right) Q_{d^{\prime}}\left(\hbar, \alpha_{i}\right) Q_{d-d^{\prime}}\left(-\hbar, \alpha_{i}\right),
\end{aligned}
$$

where

$$
Q_{d}(\hbar, x) \equiv \prod_{r=1}^{r=d} \prod_{k=1}^{k=n}\left(x-\alpha_{k}+r \hbar\right) \quad \forall d \in \overline{\mathbb{Z}}^{+}
$$

By the Residue Theorem,

$$
\begin{aligned}
\frac{1}{2 \pi \mathfrak{i}} & \oint e^{\Omega z} \frac{E_{Y, Z ; d}(\hbar, \Omega)}{\prod_{r=0}^{r=d} \prod_{k=1}^{k=n}\left(\Omega-\alpha_{k}-r \hbar\right)} d \Omega \\
= & \sum_{d^{\prime}=0}^{d^{\prime}=d} \sum_{i=1}^{i=n} e^{\left(\alpha_{i}+d^{\prime} \hbar\right) z} \frac{E_{Y, Z ; d}\left(\hbar, \alpha_{i}+d^{\prime} \hbar\right)}{\left(\prod_{k \neq i}\left(\alpha_{i}-\alpha_{k}\right)\right) Q_{d^{\prime}}\left(\hbar, \alpha_{i}\right) Q_{d-d^{\prime}}\left(-\hbar, \alpha_{i}\right)} \\
= & \sum_{d^{\prime}=0} \sum_{i=1}^{d^{\prime}=d}\left(\frac{e^{\alpha_{i} z}}{\prod_{k \neq i}\left(\alpha_{i}-\alpha_{k}\right)}\right)\left(\frac{\left(e^{\hbar z}\right)^{d^{\prime}}}{Q_{d^{\prime}}\left(\hbar, \alpha_{i}\right) Q_{d-d^{\prime}}\left(-\hbar, \alpha_{i}\right)}\right) \\
& E_{Y, Z ; d}\left(\hbar, \alpha_{i}+d^{\prime} \hbar\right) .
\end{aligned}
$$


On the other hand, since $Y, Z \in \tilde{\mathbb{Q}}_{\alpha}(\hbar, x)[[u]]$,

(2.6) $Y(\hbar, x, u)=\sum_{d=0}^{\infty} \frac{N_{Y ; d}\left(\hbar, \alpha_{i}\right)}{Q_{d}\left(\hbar, \alpha_{i}\right)} u^{d} \quad$ and $\quad Z(\hbar, x, u)=\sum_{d=0}^{\infty} \frac{N_{Z ; d}\left(\hbar, \alpha_{i}\right)}{Q_{d}\left(\hbar, \alpha_{i}\right)} u^{d}$

for unique $N_{Y ; d}, N_{Z ; d} \in \tilde{\mathbb{Q}}_{\alpha}(\hbar, x)$. By (2.2) and (2.6),

$$
\begin{aligned}
\Phi_{Y, Z}(\hbar, u, z)= & \sum_{d=0}^{\infty} \sum_{d^{\prime}=0}^{d^{\prime}=d} \sum_{i=1}^{i=n} \frac{e^{\alpha_{i} z}}{\prod_{k \neq i}\left(\alpha_{i}-\alpha_{k}\right)}\left(\frac{N_{Y ; d^{\prime}}\left(\hbar, \alpha_{i}\right)}{Q_{d^{\prime}}\left(\hbar, \alpha_{i}\right)}\right)\left(u e^{\hbar z}\right)^{d^{\prime}} \\
& \left(\frac{N_{Z ; d-d^{\prime}}\left(-\hbar, \alpha_{i}\right)}{Q_{d-d^{\prime}}\left(-\hbar, \alpha_{i}\right)}\right) u^{d-d^{\prime}} \\
= & \sum_{d=0}^{\infty} u^{d}\left(\sum_{d^{\prime}=0}^{d^{\prime}=d} \sum_{i=1}^{i=n} \frac{e^{\alpha_{i} z}}{\prod_{k \neq i}\left(\alpha_{i}-\alpha_{k}\right)}\left(\frac{\left(e^{\hbar z}\right)^{d^{\prime}}}{Q_{d^{\prime}}\left(\hbar, \alpha_{i}\right) Q_{d-d^{\prime}}\left(-\hbar, \alpha_{i}\right)}\right)\right. \\
& \left.\times N_{Y ; d^{\prime}}\left(\hbar, \alpha_{i}\right) N_{Z ; d-d^{\prime}}\left(-\hbar, \alpha_{i}\right)\right)
\end{aligned}
$$

By (2.5) and (2.7), (2.3) is satisfied if and only if

$$
\begin{array}{r}
E_{Y, Z ; d}\left(\hbar, \alpha_{i}+d^{\prime} \hbar\right)=N_{Y ; d^{\prime}}\left(\hbar, \alpha_{i}\right) \cdot N_{Z ; d-d^{\prime}}\left(-\hbar, \alpha_{i}\right) \\
\forall i=1, \ldots, n, d^{\prime}=0, \ldots, d .
\end{array}
$$

For a dense collection of complex parameters $\hbar$, there exists a unique polynomial

$$
E_{Y, Z ; d}(\hbar, \Omega) \in \tilde{\mathbb{Q}}_{\alpha}(\hbar)[\Omega]
$$

of $\Omega$-degree at most $(d+1) n-1$ that satisfies $(2.8)$.

Lemma 2.2. If $Y, Z \in \tilde{\mathbb{Q}}_{\alpha}(\hbar, x)[[u]]$ and $\left(E_{Y, Z ; d}\right)_{d \in \overline{\mathbb{Z}}^{+}} \subset \tilde{\mathbb{Q}}_{\alpha}(\hbar)[\Omega]$ are as in Lemma 2.1, then

$$
\begin{aligned}
\Phi_{Y, Z} \in \tilde{\mathbb{Q}}_{\alpha}[\hbar][[u, z]] & \Longleftrightarrow E_{Y, Z ; d} \in \tilde{\mathbb{Q}}_{\alpha}[\hbar, \Omega] \quad \forall d \in \overline{\mathbb{Z}}^{+} \\
& \Longleftrightarrow E_{Z, Y ; d} \in \tilde{\mathbb{Q}}_{\alpha}[\hbar, \Omega] \quad \forall d \in \overline{\mathbb{Z}}^{+} \\
& \Longleftrightarrow \Phi_{Z, Y} \in \tilde{\mathbb{Q}}_{\alpha}[\hbar][[u, z]] .
\end{aligned}
$$


Proof. The equivalence of the two middle statements in (2.9) follows from (2.8), which implies that

$$
E_{Z, Y ; d}(\hbar, \Omega)=E_{Y, Z ; d}(-\hbar, \Omega-d \hbar)
$$

On the other hand, by the Residue Theorem on $S^{2}$,

$$
\frac{1}{2 \pi \mathfrak{i}} \oint \frac{\Omega^{k} d \Omega}{\prod_{r=0}^{r=d} \prod_{k=1}^{k=n}\left(\Omega-\alpha_{k}-r \hbar\right)}= \begin{cases}0 & \text { if } k<(d+1) n-1, \\ 1 & \text { if } k=(d+1) n-1, \\ R_{k-(d+1) n+1}^{d}(\hbar) & \text { if } k>(d+1) n-1,\end{cases}
$$

where $R_{s}^{d} \in \mathbb{Q}_{\alpha}[\hbar]$ is given by

$$
R_{s}^{d}(\hbar)=\mathcal{D}_{w}^{s}\left(\frac{1}{\prod_{r=0}^{r=d} \prod_{k=1}^{k=n}\left(1-\left(\alpha_{k}+r \hbar\right) w\right)}\right) \forall s \in \overline{\mathbb{Z}}^{+} .
$$

The path integral in (2.10) is again taken over a simple closed loop enclosing all points $\Omega=\alpha_{k}+r \hbar$ with $r \leq d$. Write

$$
\begin{gathered}
\Phi_{Y, Z}(\hbar, u, z)=\sum_{d=0}^{\infty} \sum_{q=0}^{\infty} \frac{1}{q !} F_{d, q}(\hbar) z^{q} u^{d} \\
E_{Y, Z ; d}(\hbar, \Omega)=\sum_{s=0}^{(d+1) n-1} f_{d, s}(\hbar) \Omega^{s} .
\end{gathered}
$$

By (2.3), (2.10) and (2.11),

$$
\begin{aligned}
F_{d, q}(\hbar) & =\sum_{s=0}^{(d+1) n-1} \frac{1}{2 \pi \mathfrak{i}} \oint \frac{f_{d, s}(\hbar) \Omega^{q+s} d \Omega}{\prod_{r=0}^{r=d} \prod_{k=1}^{k=n}\left(\Omega-\alpha_{k}-r \hbar\right)} \\
& =\sum_{s=\max (0,(d+1) n-1-q)}^{(d+1) n-1} R_{q+s-(d+1) n+1}^{d}(\hbar) f_{d, s}(\alpha ; \hbar) .
\end{aligned}
$$

Since $R_{s}^{d} \in \mathbb{Q}_{\alpha}[\hbar]$, it follows that $F_{d, q} \in \tilde{\mathbb{Q}}_{\alpha}[\hbar]$ if $f_{d, s} \in \tilde{\mathbb{Q}}_{\alpha}[\hbar]$ for all $s$. Conversely, since $R_{0}^{d}(\hbar)=1$,

$$
F_{d, 0}, \ldots, F_{d,(d+1) n-1} \in \tilde{\mathbb{Q}}_{\alpha}[\hbar] \quad \Longrightarrow \quad f_{d,(d+1) n-1}, \ldots, f_{d, 0} \in \tilde{\mathbb{Q}}_{\alpha}[\hbar] .
$$

These observations imply the two remaining statements of Lemma 2.2. 
Definition 2.1. For $i, j \in[n]$ with $i \neq j$ and $d \in \mathbb{Z}^{+}$, let

$$
C_{i}^{j}(d)=\frac{\prod_{r=0}^{n d-1}\left(n \alpha_{i}+r\left(\alpha_{j}-\alpha_{i}\right) / d\right)}{d \prod_{\substack{r=1 \\(r, k) \neq(d, j)}}^{r=d} \prod_{k=1}^{k=n}\left(\alpha_{i}-\alpha_{k}+r\left(\alpha_{j}-\alpha_{i}\right) / d\right)} \in \mathbb{Q}_{\alpha} .
$$

We will call $Z \in \tilde{\mathbb{Q}}_{\alpha}(\hbar, x)[[u]] C$-recursive if

$$
\begin{aligned}
Z\left(\hbar, \alpha_{i}, u\right)= & \sum_{d=0}^{\infty}\left(\sum_{r=-N_{d}}^{r=N_{d}} Z_{i ; d}^{r} \hbar^{-r}\right) u^{d} \\
& +\sum_{d=1}^{\infty} \sum_{j \neq i} \frac{1}{\hbar-\frac{\alpha_{j}-\alpha_{i}}{d}} C_{i}^{j}(d) u^{d} Z\left(\left(\alpha_{j}-\alpha_{i}\right) / d, \alpha_{j}, u\right)
\end{aligned}
$$

for every $i \in[n]$ and for some $N_{d} \in \mathbb{Z}$ and $Z_{i ; d}^{r} \in \tilde{\mathbb{Q}}_{\alpha} \cdot{ }^{9}$

Proposition 2.1. Suppose

$$
Y, Z \in \tilde{\mathbb{Q}}_{\alpha}(\hbar, x)[[u]] \subset \tilde{\mathbb{Q}}_{\alpha}(x)\left[\left[\hbar^{-1}, u\right]\right]+\tilde{\mathbb{Q}}_{\alpha}(x)[\hbar][[u]]
$$

are such that $Z$ is $C$-recursive, $\Phi_{Y, Z} \in \tilde{\mathbb{Q}}_{\alpha}[\hbar][[u, z]]$, and for every $i \in[n]$

$$
Y\left(\hbar, \alpha_{i}, f_{i}\right) \equiv f_{i}(\bmod u)
$$

for some $f_{i} \in \tilde{\mathbb{Q}}_{\alpha}^{*}$. Then,

$Z\left(\hbar, \alpha_{i}, u\right) \equiv 0\left(\bmod \hbar^{-1}\right) \quad \forall i \in[n] \quad \Longrightarrow \quad Z\left(\hbar, \alpha_{i}, u\right)=0 \quad \forall i \in[n]$.

Remark 1. Suppose

$$
Z\left(\hbar, \alpha_{i}, u\right)=\sum_{d=0}^{\infty}\left(\sum_{r=-N_{d}}^{\infty} \tilde{Z}_{i ; d}^{r} \hbar^{-r}\right) u^{d}
$$

for some $\tilde{Z}_{i ; d}^{r} \in \tilde{\mathbb{Q}}_{\alpha}$. In the statement of Proposition 2.1 and throughout the rest of the paper,

$$
Z\left(\hbar, \alpha_{i}, u\right) \equiv \sum_{d=0}^{\infty}\left(\sum_{r=-N_{d}}^{a-1} \tilde{Z}_{i ; d^{r}}^{r}\right) u^{d}\left(\bmod \hbar^{-a}\right),
$$

i.e., we drop $\hbar^{-a}$ and higher powers of $\hbar^{-1}$, instead of higher powers of $\hbar$.

\footnotetext{
${ }^{9}$ Recursion (30.11) in [11] is a renormalization of recursion (2.13) with a slightly different coefficient $C_{i}^{j}(d)$.
} 
Remark 2. In contrast to the situation in [11, Chapter 30], the assumptions of Proposition 2.1, i.e., recursivity and $\hbar$-polynomiality with respect to $Y$, are both linear conditions on $Z$. Consequently, the $\hbar^{-1}$-term of $Z$ $\left(\hbar, \alpha_{i}, u\right)$ is no longer necessary to determine $Z$.

Proof. Suppose $d \geq 0$ and we have shown that

$$
Z\left(\hbar, \alpha_{i}, u\right) \equiv 0\left(\bmod u^{d}\right) \quad \forall i=1, \ldots, n
$$

With notation as in (2.13) and by the last assumption on $Z\left(\hbar, \alpha_{i}, u\right)$, it follows that

$$
Z\left(\hbar, \alpha_{i}, u\right) \equiv u^{d} \sum_{r=1}^{r=N_{d}} Z_{i ; d^{r}}^{r-r}\left(\bmod u^{d+1}\right) \quad \forall i=1, \ldots, n
$$

If $N_{Y ; d^{\prime}}, N_{Z ; d^{\prime}} \in \tilde{\mathbb{Q}}_{\alpha}(\hbar, x)$ are as in the proof of Lemma 2.1,

(2.16) $N_{Y ; 0}\left(\hbar, \alpha_{i}\right)=f_{i}, N_{Z ; d^{\prime}}\left(\hbar, \alpha_{i}\right)= \begin{cases}0 & \text { if } d^{\prime}<d \\ Q_{d}\left(\hbar, \alpha_{i}\right) \sum_{r=1}^{r=N_{d}} Z_{i ; d}^{r} \hbar^{-r} & \text { if } d^{\prime}=d\end{cases}$

by $(2.6),(2.14)$ and (2.15). Since

$$
E_{Z, Y ; d}\left(\hbar, \alpha_{i}+d^{\prime} \hbar\right)=0 \quad \forall d^{\prime}=0,1, \ldots, d-1, i=1, \ldots, n
$$

by $(2.8)$ and $(2.16)$ and $E_{Z, Y ; d} \in \tilde{\mathbb{Q}}_{\alpha}[\hbar, \Omega]$ by Lemma 2.2 ,

$$
E_{Z, Y ; d}(\hbar, \Omega)=\left(\prod_{d^{\prime}=0}^{d-1} \prod_{j=1}^{j=n}\left(\Omega-\alpha_{j}-d^{\prime} \hbar\right)\right) \cdot R_{d}(\hbar, \Omega)
$$

for some $R_{d} \in \tilde{\mathbb{Q}}_{\alpha}[\hbar, \Omega]$. Thus,

$$
\begin{aligned}
E_{Z, Y ; d}\left(\hbar, \alpha_{i}+d \hbar\right) & =\left(\prod_{d^{\prime}=0}^{d-1} \prod_{k=1}^{k=n}\left(\left(\alpha_{i}+d \hbar\right)-\alpha_{k}-d^{\prime} \hbar\right)\right) \cdot R_{d}\left(\hbar, \alpha_{i}+d \hbar\right) \\
& =\hbar^{d} \tilde{R}_{d}(\hbar)
\end{aligned}
$$


for some $\tilde{R}_{d} \in \tilde{\mathbb{Q}}_{\alpha}[\hbar]$. On the other hand, by (2.8) and (2.16)

$$
\begin{aligned}
E_{Z, Y ; d}\left(\hbar, \alpha_{i}+d \hbar\right) & =N_{Z ; d}\left(\hbar, \alpha_{i}\right) \cdot f_{i} \\
& =f_{i} \cdot\left(d ! \hbar^{d} \prod_{r=1}^{r=d} \prod_{k \neq i}\left(\alpha_{i}-\alpha_{k}+r \hbar\right)\right) \sum_{r=1}^{r=N_{d}} Z_{i ; d^{r}}^{r} \hbar^{-r} \\
& =f_{i} \cdot\left(d ! \prod_{r=1}^{r=d} \prod_{k \neq i}\left(\alpha_{i}-\alpha_{k}+r \hbar\right)\right) \sum_{r=1}^{r=N_{d}} Z_{i ; d}^{r} \hbar^{d-r}
\end{aligned}
$$

By (2.17) and (2.18),

$$
Z_{i ; d}^{r}=0 \quad \forall r=1, \ldots, N_{d}, i=1, \ldots, n .
$$

Along with (2.15), this implies that (2.14) holds with $d$ replaced by $d+1$.

\subsection{Admissible transforms}

This subsection is the analog of the beginning of Section 30.4 in [11]. We describe transforms of $Y, Z \in \tilde{\mathbb{Q}}_{\alpha}(\hbar, x)$ that preserve the polynomiality property of Lemma 2.2 and the recursivity property of Definition 2.1. The statement of Lemma 2.3 is followed by complete proofs. The first of the five transforms below has no analog in [11].

Lemma 2.3. Suppose $Y, Z \in \tilde{\mathbb{Q}}_{\alpha}(\hbar, x)[[u]]$ are such that $Z$ is $C$-recursive and $\Phi_{Y, Z} \in \tilde{\mathbb{Q}}_{\alpha}[\hbar][[u, z]]$. Then,

(i) if $u=e^{t}, \bar{Z} \equiv\left\{x+\hbar \frac{d}{d t}\right\} Z$ is C-recursive and $\Phi_{Y, \bar{Z}} \in \tilde{\mathbb{Q}}_{\alpha}[\hbar][[u, z]]$;

(ii) if $f \in \tilde{\mathbb{Q}}_{\alpha}[u]$, then $f Z$ is $C$-recursive and $\Phi_{Y, f Z}, \Phi_{f Y, Z} \in \tilde{\mathbb{Q}}_{\alpha}[\hbar][[u, z]]$;

(iii) if $f \in \tilde{\mathbb{Q}}_{\alpha}[\hbar]$, then $\bar{Z} \equiv f Z$ is $C$-recursive and $\Phi_{Y, \bar{Z}} \in \tilde{\mathbb{Q}}_{\alpha}[\hbar][[u, z]]$;

(iv) if $f \in \tilde{\mathbb{Q}}_{\alpha}[u], f(0)=0$ and $\bar{Y}=e^{f / \hbar} Y$, then $\bar{Z} \equiv e^{f / \hbar} Z$ is C-recursive and $\Phi_{\bar{Y}, \bar{Z}} \in \tilde{\mathbb{Q}}_{\alpha}[\hbar][[u, z]]$;

(v) if $g \in \mathbb{Q}[[u]], g(0)=0$,

$$
\bar{Y}(\hbar, x, u)=e^{x g(u) / \hbar} Y\left(\hbar, x, u e^{g(u)}\right),
$$

and

$$
\bar{Z}(\hbar, x, u)=e^{x g(u) / \hbar} Z\left(\hbar, x, u e^{g(u)}\right),
$$

then $\bar{Z}$ is $C$-recursive and $\Phi_{\bar{Y}, \bar{Z}} \in \tilde{\mathbb{Q}}_{\alpha}[\hbar][[u, z]]$. 
Remark. In fact, (ii) and (iii) are special cases of the admissible transform defined by $f \in \mathbb{Q}_{\alpha}[\hbar][[u]]$.

(i) The operator $\left\{\alpha_{i}+\hbar \frac{d}{d t}\right\}$ preserves the structure of the first term on the right-hand side of $(2.13)$. The $(d, j)$-summand in the last term becomes

$$
\begin{aligned}
& \left\{\alpha_{i}+\hbar \frac{d}{d t}\right\}\left(\frac{C_{i}^{j}(d) u^{d}}{\hbar-\frac{\alpha_{j}-\alpha_{i}}{d}} Z\left(\left(\alpha_{j}-\alpha_{i}\right) / d, \alpha_{j}, u\right)\right) \\
& \quad=\frac{C_{i}^{j}(d) u^{d}}{\hbar-\frac{\alpha_{j}-\alpha_{i}}{d}}\left\{\alpha_{i}+d \hbar+\hbar \frac{d}{d t}\right\} Z\left(\left(\alpha_{j}-\alpha_{i}\right) / d, \alpha_{j}, u\right) \\
& \quad=\frac{C_{i}^{j}(d) u^{d}}{\hbar-\frac{\alpha_{j}-\alpha_{i}}{d}} \bar{Z}\left(\left(\alpha_{j}-\alpha_{i}\right) / d, \alpha_{j}, u\right)+d C_{i}^{j}(d) u^{d} Z\left(\left(\alpha_{j}-\alpha_{i}\right) / d, \alpha_{j}, u\right) .
\end{aligned}
$$

Since the last term in (2.19) does not depend on $\hbar$ and $Z$ is $C$-recursive, it follows that $\bar{Z}$ is also $C$-recursive. Since

$$
\Phi_{\bar{Z}, Y}(\hbar, u, z)=\frac{d}{d z} \Phi_{Z, Y}(\hbar, u, z)
$$

and $\Phi_{Y, Z} \in \tilde{\mathbb{Q}}_{\alpha}[\hbar][[u, z]], \Phi_{Y, \bar{Z}} \in \tilde{\mathbb{Q}}_{\alpha}[\hbar][[u, z]]$ by the middle equivalence in Lemma 2.2.

(ii) Since $Z$ is $C$-recursive and the multiplication by $f$ preserves the structure of each of the terms in (2.13), $f Z$ is also $C$-recursive. Since $\Phi_{Y, f Z}=$ $f \Phi_{Y, Z}$ and $\Phi_{Y, Z} \in \tilde{\mathbb{Q}}_{\alpha}[\hbar][[u, z]], \Phi_{Y, f Z} \in \tilde{\mathbb{Q}}_{\alpha}[\hbar][[u, z]]$. Similarly, since $\Phi_{Z, f Y}=$ $f \Phi_{Z, Y}, \Phi_{f Y, Z} \in \tilde{\mathbb{Q}}_{\alpha}[\hbar][[u, z]]$ by Lemma 2.2 .

(iii) It is sufficient to assume that $f(\hbar)=\hbar$. The multiplication by $\hbar$ preserves the structure of the first term on the right-hand side of (2.13). The $(d, j)$-summand in the last term becomes

$$
\begin{aligned}
\hbar \frac{C_{i}^{j}(d) u^{d}}{\hbar-\frac{\alpha_{j}-\alpha_{i}}{d}} Z\left(\left(\alpha_{j}-\alpha_{i}\right) / d, \alpha_{j}, u\right)= & \frac{C_{i}^{j}(d) u^{d}}{\hbar-\frac{\alpha_{j}-\alpha_{i}}{d}} \bar{Z}\left(\left(\alpha_{j}-\alpha_{i}\right) / d, \alpha_{j}, u\right) \\
& +C_{i}^{j}(d) u^{d} Z\left(\left(\alpha_{j}-\alpha_{i}\right) / d, \alpha_{j}, u\right) .
\end{aligned}
$$

Since the last term in (2.20) does not depend on $\hbar$ and $Z$ is $C$-recursive, it follows that $\bar{Z}$ is also $C$-recursive. Since $\Phi_{Y, \bar{Z}}=-\hbar \Phi_{Y, Z}$ and $\Phi_{Y, Z} \in \tilde{\mathbb{Q}}_{\alpha}[\hbar]$ $[[u, z]], \Phi_{Y, \bar{Z}} \in \tilde{\mathbb{Q}}_{\alpha}[\hbar][[u, z]]$.

(iv) Since $f(0)=0$, i.e., $f$ contains no degree-0 term in $u$, the multiplication by $e^{f(u) / \hbar}$ preserves the structure of the first term on the right-hand 
side of (2.13). The $(d, j)$-summand in the last term becomes

$$
\begin{aligned}
e^{f(u) / \hbar} & \frac{C_{i}^{j}(d) u^{d}}{\hbar-\frac{\alpha_{j}-\alpha_{i}}{d}} Z\left(\left(\alpha_{j}-\alpha_{i}\right) / d, \alpha_{j}, u\right) \\
= & \frac{C_{i}^{j}(d) u^{d}}{\hbar-\frac{\alpha_{j}-\alpha_{i}}{d}} \bar{Z}\left(\left(\alpha_{j}-\alpha_{i}\right) / d, \alpha_{j}, u\right)+\left(e^{f(u) / \hbar}-e^{f(u) /\left(\left(\alpha_{j}-\alpha_{i}\right) / d\right)}\right) \\
& \times \frac{C_{i}^{j}(d) u^{d}}{\hbar-\frac{\alpha_{j}-\alpha_{i}}{d}} Z\left(\left(\alpha_{j}-\alpha_{i}\right) / d, \alpha_{j}, u\right) .
\end{aligned}
$$

Since $Z$ is $C$-recursive and

$$
\frac{e^{f(u) / \hbar}-e^{f(u) /\left(\left(\alpha_{j}-\alpha_{i}\right) / d\right)}}{\hbar-\frac{\alpha_{j}-\alpha_{i}}{d}} \in \tilde{\mathbb{Q}}_{\alpha}\left[\hbar, \hbar^{-1}\right][[u]],
$$

it follows that $\bar{Z}$ is $C$-recursive as well. On the other hand,

$$
\Phi_{\bar{Y}, \bar{Z}}(\hbar, u, z)=e^{\left(f\left(u e^{\hbar z}\right)-f(u)\right) / \hbar} \Phi_{Y, Z}(\hbar, u, z) .
$$

Since $\Phi_{Y, Z} \in \tilde{\mathbb{Q}}_{\alpha}[\hbar][[u, z]]$ and

$$
\left(f\left(u e^{\hbar z}\right)-f(u)\right) / \hbar \in \tilde{\mathbb{Q}}_{\alpha}[\hbar][[u, z]],
$$

(2.21) implies that $\Phi_{\bar{Y}, \bar{Z}} \in \tilde{\mathbb{Q}}_{\alpha}[\hbar][[u, z]]$ as well.

(v) Since $g(0)=0$, the operation of replacing $u$ with $u e^{g(u)}$ followed by multiplication by $e^{\alpha_{i} g(u) / \hbar}$ preserves the structure of the first term on the right-hand side of $(2.13)$. The $(d, j)$-summand in the last term becomes

$$
\begin{aligned}
& e^{\alpha_{i} g(u) / \hbar} \frac{C_{i}^{j}(d) u^{d} e^{d g(u)}}{\hbar-\frac{\alpha_{j}-\alpha_{i}}{d}} Z\left(\left(\alpha_{j}-\alpha_{i}\right) / d, \alpha_{j}, u\right) \\
& =\frac{C_{i}^{j}(d) u^{d}}{\hbar-\frac{\alpha_{j}-\alpha_{i}}{d}} \bar{Z}\left(\left(\alpha_{j}-\alpha_{i}\right) / d, \alpha_{j}, u\right) \\
& \quad+\left(e^{\left(\alpha_{i} / \hbar+d\right) g(u)}-e^{\left(\alpha_{j} /\left(\left(\alpha_{j}-\alpha_{i}\right) / d\right)\right) g(u)}\right) \frac{C_{i}^{j}(d) u^{d}}{\hbar-\frac{\alpha_{j}-\alpha_{i}}{d}} Z\left(\left(\alpha_{j}-\alpha_{i}\right) / d, \alpha_{j}, u\right) .
\end{aligned}
$$

Since $Z$ is $C$-recursive and

$$
\begin{aligned}
& \frac{e^{\left(\alpha_{i} / \hbar+d\right) g(u)}-e^{\left(\alpha_{j} /\left(\left(\alpha_{j}-\alpha_{i}\right) / d\right)\right) g(u)}}{\hbar-\frac{\alpha_{j}-\alpha_{i}}{d}} \\
& =\left.\frac{d}{\left(\alpha_{i}+d \hbar\right)-\alpha_{j}} e^{d z /\left(z-\alpha_{i}\right)}\right|_{z=\alpha_{j}} ^{z=\alpha_{i}+d \hbar} \in \mathbb{Q}_{\alpha}\left[\hbar, \hbar^{-1}\right][[u]],
\end{aligned}
$$


it follows that $\bar{Z}$ is $C$-recursive as well. On the other hand,

$$
\Phi_{\bar{Y}, \bar{Z}}(\hbar, u, z)=\Phi_{Y, Z}\left(\hbar, u e^{g(u)}, \tilde{z}\right),
$$

where

$$
\tilde{z}=z+\frac{g\left(u e^{\hbar z}\right)-g(u)}{\hbar} \in \mathbb{Q}[\hbar, z][[u]] .
$$

Since $\Phi_{Y, Z} \in \tilde{\mathbb{Q}}_{\alpha}[\hbar][[u, z]], \quad(2.22)$ implies that $\Phi_{\bar{Y}, \bar{Z}} \in \tilde{\mathbb{Q}}_{\alpha}[\hbar][[u, z]]$ as well.

\subsection{Some properties of hypergeometric series}

In this subsection we verify the three claim concerning the power series $\mathcal{Y}_{p}(\hbar, x, u)$ made in Section 1.3:

(a) $\mathcal{Y}_{-1}(\hbar, x, u)$ satisfies the $C$-recursivity condition of Definition 2.1;

(b) $\Phi_{\mathcal{Y}, \mathcal{Y}_{-1}} \in \mathbb{Q}_{\alpha}[\hbar][[u, z]]$;

(c) there exist $\tilde{C}_{p, q}^{(r)}$ as in Theorem 1.1 such that (1.21) is satisfied.

Proof (a). Note that

$$
\frac{C_{i}^{j}(d) u^{d}}{\hbar-\frac{\alpha_{j}-\alpha_{i}}{d}} \mathcal{Y}_{-1}\left(\left(\alpha_{j}-\alpha_{i}\right) / d, \alpha_{j}, u\right)=\operatorname{Res}_{z=\frac{\alpha_{j}-\alpha_{i}}{d}}\left\{\frac{1}{\hbar-z} \mathcal{Y}_{-1}\left(z, \alpha_{i}, u\right)\right\}
$$

Thus, by the Residue Theorem on $S^{2}$,

$$
\begin{aligned}
& \sum_{d=1}^{\infty} \sum_{j \neq i} \frac{C_{i}^{j}(d) u^{d}}{\hbar-\frac{\alpha_{j}-\alpha_{i}}{d}} \mathcal{Y}_{-1}\left(\left(\alpha_{j}-\alpha_{i}\right) / d, \alpha_{j}, u\right) \\
& \quad=-\operatorname{Res}_{z=\hbar, 0, \infty}\left\{\frac{1}{\hbar-z} \mathcal{Y}_{-1}\left(z, \alpha_{i}, u\right)\right\} \\
& \quad=\mathcal{Y}_{-1}\left(\hbar, \alpha_{i}, u\right)-\operatorname{Res}_{z=0, \infty}\left\{\frac{1}{\hbar-z} \mathcal{Y}_{-1}\left(z, \alpha_{i}, u\right)\right\} .
\end{aligned}
$$

On the other hand, if $\mathcal{Y}_{-1 ; d}$ is the degree- $d$ term of $\mathcal{Y}_{-1}$,

$$
\begin{aligned}
& \operatorname{Res}_{z=0}\left\{\frac{1}{\hbar-z} \mathcal{Y}_{-1 ; d}\left(z, \alpha_{i}, u\right)\right\}= \mathcal{D}_{z}^{d-1}\left\{\frac{1}{\hbar-z} \frac{\prod_{r=0}^{r=n d-1}\left(n \alpha_{i}+r z\right)}{d ! \prod_{r=1}^{r=d} \prod_{k \neq i}\left(\alpha_{i}-\alpha_{k}+r z\right)}\right\} \\
& \in \mathbb{Q}_{\alpha}\left[\hbar^{-1}\right], \\
& \operatorname{Res}_{z=\infty}\left\{\frac{1}{\hbar-z} \mathcal{Y}_{-1}\left(z, \alpha_{i}, u\right)\right\}=1,
\end{aligned}
$$


where $\mathcal{D}_{z}^{d}$ is as in (2.1). Thus, (2.23) implies that $\mathcal{Y}_{-1}$ satisfies recursion (2.13).

(b). Let

$$
R(\hbar, x, u)=\sum_{d=0}^{\infty} u^{d} \frac{\prod_{r=1}^{r=n d}(n x+r \hbar)}{\prod_{r=1}^{r=d} \prod_{k=1}^{k=n}\left(x-\alpha_{k}+r \hbar\right)}
$$

By (ii) of Lemma 2.3 , it is sufficient to show that $\Phi_{R, \mathcal{Y}_{-1}} \in \mathbb{Q}_{\alpha}[\hbar][[u, z]]$. Note that

$$
\begin{aligned}
& \frac{e^{\alpha_{i} z}}{\prod_{k \neq i}\left(\alpha_{i}-\alpha_{k}\right)} R\left(\hbar, \alpha_{i}, u e^{\hbar z}\right) \mathcal{Y}_{-1}\left(-\hbar, \alpha_{i}, u\right) \\
& \quad=\operatorname{Res}_{x=\alpha_{i}}\left\{\frac{e^{x z}}{\prod_{k=1}^{k=n}\left(x-\alpha_{k}\right)} R\left(\hbar, x, u e^{\hbar z}\right) \mathcal{Y}_{-1}(-\hbar, x, u)\right\} .
\end{aligned}
$$

Thus, by the Residue Theorem on $S^{2}$,

$$
\begin{aligned}
\Phi_{R, \mathcal{Y}_{-1}}(\hbar, u, z)= & -\operatorname{Res}_{x=\infty}\left\{\frac{e^{x z}}{\prod_{k=1}^{k=n}\left(x-\alpha_{k}\right)} R\left(\hbar, x, u e^{\hbar z}\right) \mathcal{Y}_{-1}(-\hbar, x, u)\right\} \\
= & \sum_{p=0}^{\infty} \frac{z^{n-1+p}}{(n-1+p) !} \mathcal{D}_{w}^{p} \\
& \left\{\frac{1}{\prod_{k \neq i}\left(1-\alpha_{k} w\right)}\left(\sum_{d=0}^{\infty} u^{d} e^{d \hbar z} \frac{\prod_{r=1}^{r=n d}(n+r \hbar w)}{\prod_{r=1}^{r=d} \prod_{k=1}^{k=n}\left(1-\left(\alpha_{k}-r \hbar\right) w\right)}\right)\right. \\
& \left.\times\left(\sum_{d=0}^{\infty} u^{d} \frac{\prod_{r=0}^{r=n d-1}(n-r \hbar w)}{\prod_{r=1}^{r=d} \prod_{k=1}^{k=n}\left(1-\left(\alpha_{k}+r \hbar\right) w\right)}\right)\right\} .
\end{aligned}
$$

The $p$ th summand above is polynomial in $\hbar$. Thus, $\Phi_{R, \mathcal{Y}_{-1}} \in \mathbb{Q}_{\alpha}[\hbar][[u, z]]$.

(c). Suppose $p \in \mathbb{Z}^{+}$, and we have constructed power series

$$
\mathcal{Y}_{0}, \mathcal{Y}_{1}, \ldots, \mathcal{Y}_{p-1} \in \mathbb{Q}_{\alpha}(\hbar, x)\left[\left[e^{t}\right]\right]
$$


satisfying the second equation in (1.16) and (1.21), so that

$$
e^{x t / \hbar} \mathcal{Y}_{p-1}\left(\hbar, x, e^{t}\right)=x^{p}+\sum_{q=1}^{\infty}\left(\sum_{r=0}^{p-1+q} C_{p-1, q}^{(r)}(t) x^{p+q-r}\right) \hbar^{-q}
$$

where $C_{p-1, q}^{(r)}(t)$ is a degree- $r$ symmetric polynomial in $\alpha_{1}, \ldots, \alpha_{n}$ with coefficients in $\mathbb{Q}[t]\left[\left[e^{t}\right]\right]$ such that

$$
C_{p-1, q}^{(0)}(t)=I_{p-1, p-1+q}(t) / I_{p-1, p-1}(t)
$$

This assumption is satisfied for $p=1$ by (1.12) and (1.13). Since $\mathcal{Y}_{p-1} \in$ $\mathbb{Q}_{\alpha}(\hbar, x)\left[\left[e^{t}\right]\right]$,

$$
\begin{aligned}
& C_{p-1,1}^{(0)}(t) \in t+\mathbb{Q}_{\alpha}\left[\left[e^{t}\right]\right], \quad C_{p-1,1}^{(r)}(t) \in \mathbb{Q}_{\alpha}\left[\left[e^{t}\right]\right] \quad \forall r \geq 1 \\
& \Longrightarrow \frac{d}{d t} C_{p-1,1}^{(r)}(t) \in \mathbb{Q}_{\alpha}\left[\left[e^{t}\right]\right] \quad \forall r \geq 0 .
\end{aligned}
$$

Thus,

$$
\begin{aligned}
\mathcal{Y}_{p}\left(\hbar, x, e^{t}\right) \equiv & e^{-x t / \hbar} \frac{\hbar}{I_{p, p}(t)} \frac{d}{d t}\left(e^{x t / \hbar} \mathcal{Y}_{p-1}\left(\hbar, x, e^{t}\right)\right) \\
& -\frac{1}{I_{p, p}(t)} \sum_{r=1}^{r=p}\left(\frac{d}{d t} C_{p-1,1}^{(r)}(t)\right) \mathcal{Y}_{p-r}\left(\hbar, x, e^{t}\right) \in \mathbb{Q}_{\alpha}(\hbar, x)\left[\left[e^{t}\right]\right] .
\end{aligned}
$$

By (1.9), (2.24) and (2.25) are satisfied with $p$ replaced by $p+1$ and

$$
C_{p, q}^{(r)}(t)=\frac{1}{I_{p, p}(t)} \frac{d}{d t} C_{p-1, q+1}^{(r)}(t)-\frac{1}{I_{p, p}(t)} \sum_{s=1}^{\min (p, r)}\left(\frac{d}{d t} C_{p-1,1}^{(s)}(t)\right) C_{p-s, q}^{(r-s)}(t)
$$

In particular, $\mathcal{Y}_{p}$ satisfies (1.21). By (2.27), the coefficients $\tilde{C}_{p, q}^{(r)}$ are inductively defined by

$$
\begin{aligned}
\tilde{C}_{p, q}^{(r)}\left(e^{t}\right)= & I_{p, p}(t)^{-1} \\
& \left(\frac{d}{d t} \tilde{C}_{p-1, q}^{(r)}+I_{q, q}(t) \tilde{C}_{p-1, q-1}^{(r)}\left(e^{t}\right)-\sum_{s=1}^{r-1}\left(\frac{d}{d t} C_{p-1,1}^{(s)}\right) \tilde{C}_{p-s, q}^{(q-s)}\right),
\end{aligned}
$$

where $\quad \tilde{C}_{p-1, p-r}^{(r)} \equiv-C_{p-1,1}^{(r)}, \quad \tilde{C}_{p-1,-1}^{(r)} \equiv 0, \quad \tilde{C}_{p^{\prime}, q}^{(r)} \equiv 0 \forall r \leq 0$. 
Thus, by (2.26) and inductive assumptions, $\tilde{C}_{p, q}^{(r)} \in \mathbb{Q}_{\alpha}\left[\left[e^{t}\right]\right]$ is a degree- $r$ symmetric polynomial in $\alpha_{1}, \ldots, \alpha_{n}$ with coefficients in $\mathbb{Q}_{\alpha}\left[\left[e^{t}\right]\right]$, as required.

\section{Localization computations}

\subsection{Equivariant cohomology}

In Section 3.2, we apply the classical localization theorem (3.9) with the standard action of the $n$-torus $\mathbb{T}$ on $\mathbb{P}^{n-1}$ and on $\overline{\mathfrak{M}}_{0, m}\left(\mathbb{P}^{n-1}, d\right)$ to verify Lemma 1.1. In Section 3.3, we apply (3.9) with an action of the $(n+1)$-torus

$$
\widetilde{\mathbb{T}} \equiv \mathbb{T} \times \mathbb{T}^{1}
$$

on $\mathbb{P}^{n-1} \times \mathbb{P}^{1}$ and on a subspace of $\overline{\mathfrak{M}}_{0, m}\left(\mathbb{P}^{1} \times \mathbb{P}^{n-1},(1, d)\right)$ to verify Lemma 1.2. The aim of this subsection is to review the basics of equivariant cohomology and to set notation. Throughout this subsection, $G$ denotes an $m$-torus, either $\left(\mathbb{C}^{*}\right)^{m}$ or $\left(S^{1}\right)^{m}$.

The $m$-torus $G$ acts freely on $E G=\left(\mathbb{C}^{\infty}\right)^{m}-0\left(\right.$ or $\left.\left(S^{\infty}\right)^{m}\right)$ :

$$
\left(e^{\mathfrak{i} \theta_{1}}, \ldots, e^{\mathfrak{i} \theta_{m}}\right) \cdot\left(z_{1}, \ldots, z_{m}\right)=\left(e^{\mathfrak{i} \theta_{1}} z_{1}, \ldots, e^{\mathfrak{i} \theta_{m}} z_{m}\right)
$$

Thus, the classifying space for $G$ and its group cohomology are given by

$$
B G \equiv E G / G=\left(\mathbb{P}^{\infty}\right)^{m} \quad \text { and } \quad H_{G}^{*} \equiv H^{*}(B G ; \mathbb{Q})=\mathbb{Q}\left[\alpha_{1}, \ldots, \alpha_{m}\right],
$$

where $\alpha_{i}=\pi_{i}^{*} c_{1}\left(\gamma^{*}\right)$ if

$$
\pi_{i}:\left(\mathbb{P}^{\infty}\right)^{m} \longrightarrow \mathbb{P}^{\infty} \text { and } \gamma \longrightarrow \mathbb{P}^{\infty}
$$

are the projection onto the $i$ th component and the tautological line bundle, respectively. Denote by $\mathcal{H}_{G}^{*}$ the field of fractions of $H_{G}^{*}$ :

$$
\mathcal{H}_{G}^{*}=\mathbb{Q}_{\alpha} \equiv \mathbb{Q}\left(\alpha_{1}, \ldots, \alpha_{m}\right) .
$$

A representation $\rho$ of $G$, i.e., a linear action of $G$ on $\mathbb{C}^{k}$, induces a vector bundle over $B G$ :

$$
V_{\rho} \equiv E G \times_{G} \mathbb{C}^{k} .
$$

If $\rho$ is one dimensional, we will call

$$
c_{1}\left(V_{\rho}^{*}\right)=-c_{1}\left(V_{\rho}\right) \in H_{G}^{*} \subset \mathcal{H}_{G}^{*}
$$


the weight of $\rho$. For example, $\alpha_{i}$ is the weight of representation

$$
\pi_{i}: G \longrightarrow \mathbb{C}^{*}, \quad\left(e^{\mathrm{i} \theta_{1}}, \ldots, e^{\mathrm{i} \theta_{m}}\right) \cdot z=e^{\mathrm{i} \theta_{i}} z .
$$

More generally, if a representation $\rho$ of $G$ on $\mathbb{C}^{k}$ splits into one-dimensional representations with weights $\beta_{1}, \ldots, \beta_{k}$, we will call $\beta_{1}, \ldots, \beta_{k}$ the weights of $\rho$. In such a case,

$$
e\left(V_{\rho}^{*}\right)=\beta_{1} \cdot \ldots \cdot \beta_{k} .
$$

We will call the representation $\rho$ of $\mathbb{T}$ on $\mathbb{C}^{n}$ with weights $\alpha_{1}, \ldots, \alpha_{n}$ the standard representation of $\mathbb{T}$.

If $G$ acts on a topological space $M$, let

$$
H_{G}^{*}(M) \equiv H^{*}(B G ; \mathbb{Q}), \quad \text { where } \quad B M=E G \times_{G} M,
$$

be the equivariant cohomology of $M$. The projection map $B M \longrightarrow B G$ induces an action of $H_{G}^{*}$ on $H_{G}^{*}(M)$. Let

$$
\mathcal{H}_{G}^{*}(M)=H_{G}^{*}(M) \otimes_{H_{G}^{*}} \mathcal{H}_{G}^{*}
$$

If the $G$-action on $M$ lifts to an action on a (complex) vector bundle $V \longrightarrow$ $M$, then

$$
B V \equiv E G \times_{G} V
$$

is a vector bundle over $B M$. Let

$$
\mathbf{e}(V) \equiv e(B V) \in H_{G}^{*}(M) \subset \mathcal{H}_{G}^{*}(M)
$$

denote the equivariant Euler class of $V$.

The standard action of $\mathbb{T}$ on $\mathbb{P}^{n-1}$ is the action induced by the standard action $\rho$ of $\mathbb{T}$ on $\mathbb{C}^{n}$ :

$$
\left(e^{\mathrm{i} \theta_{1}}, \ldots, e^{\mathrm{i} \theta_{n}}\right) \cdot\left[z_{1}, \ldots, z_{n}\right]=\left[e^{\mathrm{i} \theta_{1}} z_{1}, \ldots, e^{\mathrm{i} \theta_{n}} z_{n}\right] .
$$

Since $B \mathbb{P}^{n-1}=\mathbb{P} V_{\rho}$,

$$
\begin{aligned}
H_{\mathbb{T}}^{*}\left(\mathbb{P}^{n-1}\right) & \equiv H^{*}\left(\mathbb{P} V_{\rho} ; \mathbb{Q}\right) \\
& =\mathbb{Q}\left[x, \alpha_{1}, \ldots, \alpha_{n}\right] /\left(x^{n}+c_{1}\left(V_{\rho}\right) x^{n-1}+\ldots+c_{n}\left(V_{\rho}\right)\right),
\end{aligned}
$$

where $x=c_{1}\left(\tilde{\gamma}^{*}\right)$ and $\tilde{\gamma} \longrightarrow \mathbb{P} V_{\rho}$ is the tautological line bundle. Since

$$
c\left(V_{\rho}\right)=\left(1-\alpha_{1}\right) \ldots\left(1-\alpha_{n}\right)
$$


it follows that

$$
H_{\mathbb{T}}^{*}\left(\mathbb{P}^{n-1}\right)=\mathbb{Q}\left[x, \alpha_{1}, \ldots, \alpha_{n}\right] /\left(x-\alpha_{1}\right) \ldots\left(x-\alpha_{n}\right)
$$

and

$$
\mathcal{H}_{\mathbb{T}}^{*}\left(\mathbb{P}^{n-1}\right)=\mathbb{Q}_{\alpha}[x] /\left(x-\alpha_{1}\right) \ldots\left(x-\alpha_{n}\right) .
$$

The standard action of $\mathbb{T}$ on $\mathbb{P}^{n-1}$ has $n$-fixed points:

$$
P_{1}=[1,0, \ldots, 0], \quad P_{2}=[0,1,0, \ldots, 0], \quad \ldots \quad P_{n}=[0, \ldots, 0,1] .
$$

For each $i=1,2, \ldots, n$, let

$$
\phi_{i}=\prod_{k \neq i}\left(x-\alpha_{k}\right) \in H_{\mathbb{T}}^{*}\left(\mathbb{P}^{n-1}\right)
$$

By Equation (3.10) below, $\phi_{i}$ is the equivariant Poincare dual of $P_{i}$. We also note that $\left.\tilde{\gamma}\right|_{B P_{i}}=V_{\pi_{i}}$, where $\pi_{i}$ is as in (3.1). Thus, the restriction map on the equivariant cohomology induced by the inclusion $P_{i} \longrightarrow \mathbb{P}^{n-1}$ is given by

$$
\begin{aligned}
H_{\mathbb{T}}^{*}\left(\mathbb{P}^{n-1}\right)= & \mathbb{Q}\left[x, \alpha_{1}, \ldots, \alpha_{n}\right] / \prod_{k=1}^{k=n}\left(x-\alpha_{k}\right) \longrightarrow H_{\mathbb{T}}^{*}\left(P_{i}\right)=\mathbb{Q}\left[\alpha_{1}, \ldots, \alpha_{n}\right], \\
& x \longrightarrow \alpha_{i} .
\end{aligned}
$$

By (3.5),

$$
\eta=\left.0 \in H_{\mathbb{T}}^{*}\left(\mathbb{P}^{n-1}\right) \quad \Longleftrightarrow \quad \eta\right|_{P_{i}}=0 \in H_{\mathbb{T}}^{*} \quad \forall i=1,2, \ldots, n .
$$

The tautological line bundle $\gamma_{n-1} \longrightarrow \mathbb{P}^{n-1}$ is a subbundle of $\mathbb{P}^{n-1} \times$ $\mathbb{C}^{n}$ preserved by the diagonal action of $\mathbb{T}$. Thus, the action of $\mathbb{T}$ on $\mathbb{P}^{n-1}$ naturally lifts to an action on $\gamma_{n-1}$ and

$$
\left.\mathbf{e}\left(\gamma_{n-1}^{*}\right)\right|_{P_{i}}=\alpha_{i} \quad \forall i=1,2, \ldots, n
$$

Via the exact sequence

$$
0 \longrightarrow \gamma_{n-1}^{*} \otimes \gamma_{n-1} \longrightarrow \gamma_{n-1}^{*} \otimes\left(\mathbb{P}^{n-1} \times \mathbb{C}^{n}\right) \longrightarrow T \mathbb{P}^{n-1} \longrightarrow 0
$$


of vector bundles on $\mathbb{P}^{n-1}, \mathbb{T}$ also lifts to an action on $T \mathbb{P}^{n-1}$. By (3.2) and (3.7),

$$
\left.\mathbf{e}\left(T \mathbb{P}^{n-1}\right)\right|_{P_{i}}=\prod_{k \neq i}\left(\alpha_{i}-\alpha_{k}\right)=\left.\phi_{i}\right|_{P_{i}} \quad \forall i=1,2, \ldots, n .
$$

If $G$ acts smoothly on a smooth compact oriented manifold $M$, there is a well-defined integration-along-the-fiber homomorphism

$$
\int_{M}: H_{G}^{*}(M) \longrightarrow H_{G}^{*}
$$

for the fiber bundle $B M \longrightarrow B G$. The classical localization theorem of [1] relates it to integration along the fixed locus of the $G$-action. The latter is a union of smooth compact orientable manifolds $F$ and $G$ acts on the normal bundle $\mathcal{N} F$ of each $F$. Once an orientation of $F$ is chosen, there is a well-defined integration-along-the-fiber homomorphism

$$
\int_{F}: H_{G}^{*}(F) \longrightarrow H_{G}^{*}
$$

The localization theorem states that

$$
\int_{M} \psi=\sum_{F} \int_{F} \frac{\left.\psi\right|_{F}}{\mathbf{e}(\mathcal{N} F)} \in \mathcal{H}_{G}^{*} \quad \forall \psi \in H_{G}^{*}(M),
$$

where the sum is taken over all components $F$ of the fixed locus of $G$. Part of the statement of $(3.9)$ is that $\mathbf{e}(\mathcal{N} F)$ is invertible in $\mathcal{H}_{G}^{*}(F)$. In the case of the standard action of $\mathbb{T}$ on $\mathbb{P}^{n-1},(3.9)$ implies that

$$
\left.\eta\right|_{P_{i}}=\int_{\mathbb{P}^{n-1}} \eta \phi_{i} \in \mathcal{H}_{\mathbb{T}} \quad \forall \eta \in \mathcal{H}_{\mathbb{T}}^{*}\left(\mathbb{P}^{n-1}\right), i=1,2, \ldots, n ;
$$

see also (3.8).

Finally, if $f: M \longrightarrow M^{\prime}$ is a $G$-equivariant map between two compact oriented manifolds, there is a well-defined pushforward homomorphism

$$
f_{*}: H_{G}^{*}(M) \longrightarrow H_{G}^{*}\left(M^{\prime}\right) \text {. }
$$

It is characterized by the property that

$$
\int_{M^{\prime}}\left(f_{*} \eta\right) \psi=\int_{M} \eta\left(f^{*} \psi\right) \quad \forall \eta \in H_{G}^{*}(M), \psi \in H_{G}^{*}\left(M^{\prime}\right) .
$$


The homomorphism $\int_{M}$ of the previous paragraph corresponds to $M^{\prime}$ being a point. It is immediate from (3.11) that

$$
f_{*}\left(\eta\left(f^{*} \psi\right)\right)=\left(f_{*} \eta\right) \psi \quad \forall \eta \in H_{G}^{*}(M), \psi \in H_{G}^{*}\left(M^{\prime}\right) .
$$

\subsection{Proof of Lemma 1.1}

The standard $\mathbb{T}$-action on $\mathbb{P}^{n-1}$ (as well as any other action) induces $\mathbb{T}$ actions on moduli spaces of stable maps $\overline{\mathfrak{M}}_{0, m}\left(\mathbb{P}^{n-1}, d\right)$ by composition on the right:

$$
g \cdot[\mathcal{C}, f]=[\mathcal{C}, g \circ f] \quad \forall g \in \mathbb{T},[\mathcal{C}, f] \in \overline{\mathfrak{M}}_{0, m}\left(\mathbb{P}^{n-1}, d\right) .
$$

All evaluation maps

$$
\mathrm{ev}_{i}: \overline{\mathfrak{M}}_{0, m}\left(\mathbb{P}^{n-1}, d\right) \longrightarrow \mathbb{P}^{n-1}, \quad\left[\mathcal{C}, y_{1}, \ldots, y_{k}, f\right] \longrightarrow f\left(y_{i}\right),
$$

are $\mathbb{T}$-equivariant. Via the natural lift of the $\mathbb{T}$-action to $\gamma_{n-1}^{*} \longrightarrow \mathbb{P}^{n-1}$ described in Section 3.1, the $\mathbb{T}$-action on $\overline{\mathfrak{M}}_{0, m}\left(\mathbb{P}^{n-1}, d\right)$ lifts to $\mathbb{T}$-actions on the vector bundles $\mathcal{V}_{0}, \mathcal{V}_{0}^{\prime}$ and $\mathcal{V}_{0}^{\prime \prime}$, as well as on the universal tangent line bundles.

As described in detail in [11, Section 27.3], the fixed loci of the $\mathbb{T}$-action on $\overline{\mathfrak{M}}_{0, m}\left(\mathbb{P}^{n-1}, d\right)$ are indexed by decorated graphs that have no loops. A graph consists of a set Ver of vertices and a collection Edg of edges, i.e., of two-element subsets of Ver. A loop in a graph (Ver, Edg) is a subset of Edg of the form

$$
\left\{\left\{v_{1}, v_{2}\right\},\left\{v_{2}, v_{3}\right\}, \ldots,\left\{v_{N}, v_{1}\right\}\right\}, \quad v_{1}, \ldots, v_{N} \in \text { Ver, } N \geq 1 .
$$

Neither of the three graphs in Figure 1 has a loop. A decorated graph is a tuple

$$
\Gamma=(\text { Ver, } \operatorname{Edg} ; \mu, \mathfrak{d}, \eta),
$$

where (Ver, Edg) is a graph and

$$
\mu: \text { Ver } \longrightarrow[n], \quad \mathfrak{d}: \operatorname{Edg} \longrightarrow \mathbb{Z}^{+}, \quad \text { and } \quad \eta:[m] \longrightarrow \text { Ver }
$$

are maps such that

$$
\mu\left(v_{1}\right) \neq \mu\left(v_{2}\right) \quad \text { if } \quad\left\{v_{1}, v_{2}\right\} \in \text { Edg. }
$$



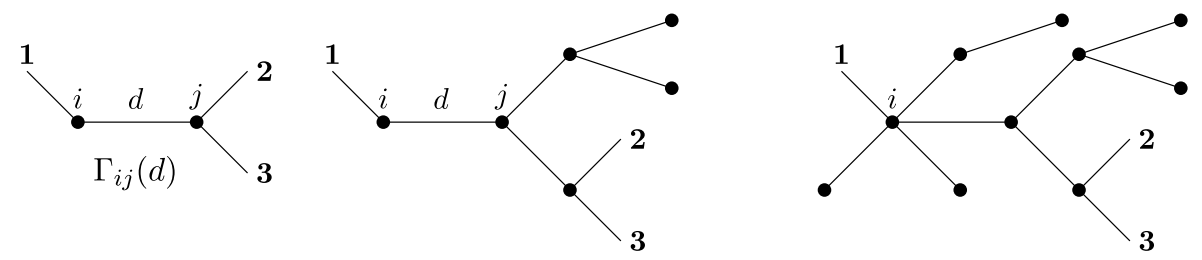

Figure 1: Two graphs of type $A_{i}(j ; d) \subset A_{i}$ and a graph of type $B_{i}$.

In Figure 1, the values of the map $\mu$ on some of the vertices are indicated by letters next to those vertices. Similarly, the value of the map $\mathfrak{d}$ on one of the edges is indicated by a letter next to the edge. The three elements of the set $[m]=[3]$ are shown in bold face. They are linked by line segments to their images under $\eta$. By (3.14), no two consecutive vertex labels are the same and thus $j \neq i$.

The fixed locus $\mathcal{Z}_{\Gamma}$ of $\overline{\mathfrak{M}}_{0, m}\left(\mathbb{P}^{n-1}, d\right)$ corresponding to a decorated graph $\Gamma$ consists of the stable maps $f$ from a genus-zero nodal curve $\mathcal{C}_{f}$ with $m$ marked points into $\mathbb{P}^{n-1}$ that satisfy the following conditions. The components of $\mathcal{C}_{f}$ on which the map $f$ is not constant are rational and correspond to the edges of $\Gamma$. Furthermore, if $e=\left\{v_{1}, v_{2}\right\}$ is an edge, the restriction of $f$ to the component $\mathcal{C}_{f, e}$ corresponding to $e$ is a degree-d $(e)$ cover of the line

$$
\mathbb{P}_{\mu\left(v_{1}\right), \mu\left(v_{2}\right)}^{1} \subset \mathbb{P}^{n-1}
$$

passing through the fixed points $P_{\mu\left(v_{1}\right)}$ and $P_{\mu\left(v_{2}\right)}$. The map $\left.u\right|_{\mathcal{C}_{f, e}}$ is ramified only over $P_{\mu\left(v_{1}\right)}$ and $P_{\mu\left(v_{2}\right)}$. In particular, $\left.f\right|_{\mathcal{C}_{f, e}}$ is unique up to isomorphism. The remaining, contracted, components of $\mathcal{C}_{f}$ are indexed by the vertices $v \in$ Ver such that

$$
\operatorname{val}(v) \equiv\left|\left\{v^{\prime} \in \operatorname{Ver}:\left\{v, v^{\prime}\right\} \in \operatorname{Edg}\right\}\right|+|\{i \in[m]: \eta(i)=v\}| \geq 3
$$

The map $f$ takes such a component $\mathcal{C}_{f, v}$ to the fixed point $\mu(v)$. Thus,

$$
\mathcal{Z}_{\Gamma} \approx \overline{\mathcal{M}}_{\Gamma} \equiv \prod_{v \in \operatorname{Ver}} \overline{\mathcal{M}}_{0, \operatorname{val}(v)}
$$

where $\overline{\mathcal{M}}_{0, l}$ denotes the moduli space of stable genus-zero curves with $l$ marked points. For the purposes of this definition, $\overline{\mathcal{M}}_{0,1}$ and $\overline{\mathcal{M}}_{0,2}$ are 
one-point spaces. For example, in the case of the last diagram in Figure 1,

$$
\mathcal{Z}_{\Gamma} \approx \overline{\mathcal{M}}_{\Gamma} \equiv \overline{\mathcal{M}}_{0,5} \times \overline{\mathcal{M}}_{0,3}^{3} \times \overline{\mathcal{M}}_{0,2} \times \overline{\mathcal{M}}_{0,1}^{5} \approx \overline{\mathcal{M}}_{0,5}
$$

is a fixed locus ${ }^{10}$ in $\overline{\mathfrak{M}}_{0,3}\left(\mathbb{P}^{n-1}, d\right)$ for some $d \geq 9$.

We now verify Lemma 1.1. Let

$$
\eta^{\beta}=\prod_{j=2}^{j=m}\left(\psi_{j}^{\beta_{j}} \mathrm{ev}_{j}^{*} \eta_{j}\right)
$$

Suppose $\Gamma$ is a decorated graph as in (3.13) that contributes to (1.19), in the sense of the localization formula (3.9). By (3.4) and (3.5),

$$
\mathrm{ev}_{1}^{*} \phi_{i}=\prod_{k \neq i}\left(\alpha_{\mu(\eta(1))}-\alpha_{k}\right)=\delta_{i, \mu(\eta(1))} \prod_{k \neq i}\left(\alpha_{i}-\alpha_{k}\right),
$$

where $\delta_{i, \mu(\eta(1))}$ is the Kronecker delta function. Thus, by (3.9), $\Gamma$ does not contribute to (1.4) unless $\mu(\eta(1))=i$, i.e., the marked point 1 of the map is taken to the point $P_{i} \in \mathbb{P}^{n-1}$. There are two types of graphs that do (or may) contribute to (1.4); they will be called $A_{i}$-and $B_{i}$-types. In a graph of the $A_{i}$-type, the marked point 1 is attached to a vertex $v_{0} \in$ Ver of valence two which is labeled $i$. In a graph of the $B_{i}$-type, the marked point 1 is attached to a vertex $v_{0}$ of valence at 3 , which is still labeled $i$. Examples of the two types are depicted in Figure 1.

Suppose $\Gamma$ is a graph of type $B_{i}$ and

$$
\mathcal{Z}_{\Gamma} \subset \overline{\mathfrak{M}}_{0, m}\left(\mathbb{P}^{n-1}, d\right)
$$

so that $\Gamma$ contributes to the coefficient of $u^{d}$ in (1.4). In this case, the restriction of $\psi_{1}$ to $\mathcal{Z}_{\Gamma}$ is the pull-back of a $\psi$-class from the component $\overline{\mathcal{M}}_{0, \operatorname{val}\left(v_{0}\right)}$ in decomposition (3.15). Since the $\mathbb{T}$-action on the corresponding tautological line bundle is trivial,

$$
\left.\psi_{1}^{k}\right|_{\mathcal{Z}_{\Gamma}}=0 \quad \forall k \geq d+m>\operatorname{val}\left(v_{0}\right)-3 .
$$

Thus, $\Gamma$ contributes a polynomial in $\hbar^{-1}$, of degree at most $d+m$, to the coefficient of $u^{d}$ in (1.4). Therefore, the contributions of the loci of type $B_{i}$ to (1.4) are accounted for by the middle term in (2.13).

\footnotetext{
${ }^{10}$ after dividing by an appropriate automorphism group; see [11, Section 27.3]
} 

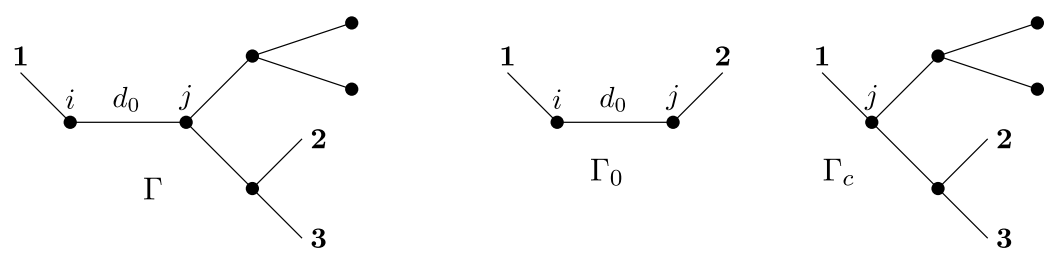

Figure 2: A graph of type $A_{i}^{*}\left(j ; d_{0}\right)$ and its two subgraphs.

A graph $\Gamma$ as in (3.13) of type $A_{i}$ has a unique vertex $v$ joined to $v_{0}$. Denote by $A_{i}\left(j ; d_{0}\right)$ the set of all graphs $\Gamma$ of type $A_{i}$ such that $\mu(v)=j$ and $\mathfrak{d}\left(\left\{v_{0}, v\right\}\right)=d_{0}$, i.e., the unique vertex $v$ of $\Gamma$ joined to $v_{0}$ is mapped to $P_{j} \in \mathbb{P}^{n-1}$ and the edge $\left\{v_{0}, v\right\}$ corresponds to the $d_{0}$-fold cover of $\mathbb{P}_{i j}^{1}$ branched only over $P_{i}$ and $P_{j}$. By (3.14),

$$
A_{i}=\bigcup_{d_{0}=1}^{\infty} \bigcup_{j \neq i} A_{i}\left(j ; d_{0}\right) .
$$

Suppose $\Gamma \in A_{i}\left(j ; d_{0}\right)$ and $v$ is the unique vertex joined to $v_{0}$ by an edge. We break $\Gamma$ at $v$ into two graphs:

(i) $\Gamma_{0}$ consisting of the vertices $v_{0}$ and $v$, the edge $\left\{v_{0}, v\right\}$, and marked points 1 and 2 attached to $v_{0}$ and $v$, respectively;

(ii) $\Gamma_{c}$ consisting all vertices and edges of $\Gamma$, other than the vertex $v_{0}$ and the edge $\left\{v_{0}, v\right\}$, with a new marked point attached to $v$;

see Figure 2. Let $d_{c}$ denote the degree of $\Gamma_{c}$, i.e., the sum of all edge labels. By (3.15),

$$
\mathcal{Z}_{\Gamma} \approx \mathcal{Z}_{\Gamma_{0}} \times \mathcal{Z}_{\Gamma_{c}}
$$

Denote by $\pi_{0}$ and $\pi_{c}$ the two component projection maps.

By Section 27.4 in [11],

$$
\begin{aligned}
\left.\mathcal{V}_{0}^{\prime \prime}\right|_{\mathcal{Z}_{\Gamma}} & =\pi_{0}^{*} \mathcal{V}_{0}^{\prime \prime} \oplus \pi_{c}^{*} \mathcal{V}_{0}^{\prime \prime}, \\
\frac{\mathcal{N} \mathcal{Z}_{\Gamma}}{T_{P_{i}} \mathbb{P}^{n-1}} & =\pi_{0}^{*}\left(\frac{\mathcal{N} \mathcal{Z}_{\Gamma_{0}}}{T_{P_{i}} \mathbb{P}^{n-1}}\right) \oplus \pi_{c}^{*}\left(\frac{\mathcal{N} \mathcal{Z}_{\Gamma_{c}}}{T_{P_{j}} \mathbb{P}^{n-1}}\right) \oplus \pi_{0}^{*} L_{2} \otimes \pi_{c}^{*} L_{1},
\end{aligned}
$$


where $L_{2} \longrightarrow \mathcal{Z}_{\Gamma_{0}}$ and $L_{1} \longrightarrow \mathcal{Z}_{\Gamma_{c}}$ are the tautological tangent line bundles. Thus, by (3.8),

$$
\begin{aligned}
\left.\frac{\mathbf{e}\left(\mathcal{V}_{0}^{\prime \prime}\right) \eta^{\beta}}{\hbar-\psi_{1}}\right|_{\mathcal{Z}_{\Gamma}} & =\pi_{0}^{*}\left(\frac{\mathbf{e}\left(\mathcal{V}_{0}^{\prime \prime}\right)}{\hbar-\psi_{1}}\right) \cdot \pi_{c}^{*}\left(\mathbf{e}\left(\mathcal{V}_{0}^{\prime \prime}\right) \eta^{\beta}\right) \\
\frac{\mathrm{ev}_{1}^{*} \phi_{i} \mid \mathcal{Z}_{\Gamma}}{\mathbf{e}\left(N \mathcal{Z}_{\Gamma}\right)} & =\pi_{0}^{*}\left(\frac{\mathrm{ev}_{1}^{*} \phi_{i}}{\mathbf{e}\left(\mathcal{N} \mathcal{Z}_{\Gamma_{0}}\right)}\right) \cdot \pi_{c}^{*}\left(\frac{\mathrm{ev}_{1}^{*} \phi_{j}}{\mathbf{e}\left(\mathcal{N} \mathcal{Z}_{\Gamma_{0}}\right)}\right) \cdot \frac{1}{\pi_{0}^{*} c_{1}\left(L_{2}\right)-\pi_{c}^{*} \psi_{1}}
\end{aligned}
$$

By Sections 27.1 and 27.2 in [11], on $\mathcal{Z}_{\Gamma_{0}}$

$$
\begin{gathered}
\mathbf{e}\left(\mathcal{V}_{0}^{\prime \prime}\right)=\prod_{r=0}^{n d_{0}-1}\left(n \alpha_{i}+r \frac{\alpha_{j}-\alpha_{i}}{d_{0}}\right), \quad \psi_{1}=c_{1}\left(L_{2}\right)=\frac{\alpha_{j}-\alpha_{i}}{d_{0}} \\
\mathbf{e}\left(N \mathcal{Z}_{\Gamma_{0}}\right)=(-1)^{d_{0}} \prod_{r=1}^{r=d_{0}}\left(r \frac{\alpha_{j}-\alpha_{i}}{d_{0}}\right)^{2} \prod_{r=0}^{r=d_{0}} \prod_{k \neq i, j}\left(\alpha_{i}-\alpha_{k}+r \frac{\alpha_{j}-\alpha_{i}}{d_{0}}\right) .
\end{gathered}
$$

Thus, using (3.8) and taking into the account the automorphism group, $\mathbb{Z}_{d_{0}}$, we obtain

$$
\int_{\mathcal{Z}_{\Gamma_{0}}} \frac{\mathbf{e}\left(\mathcal{V}_{0}^{\prime \prime}\right) \mathrm{ev}_{1}^{*} \phi_{i}}{\left(\hbar-\psi_{1}\right) \mathbf{e}\left(N \mathcal{Z}_{\Gamma_{0}}\right)}=\frac{1}{\hbar-\frac{\alpha_{j}-\alpha_{i}}{d_{0}}} C_{i}^{j}\left(d_{0}\right)
$$

By (3.18), (3.21) and (3.22), the contribution of $\Gamma$ to (1.19) is

$$
\begin{aligned}
& \left.u^{d_{0}+d_{c}} \int_{\mathcal{Z}_{\Gamma}} \frac{\mathbf{e}\left(\mathcal{V}_{0}^{\prime \prime}\right) \mathrm{ev}_{1}^{*} \phi_{i} \eta^{\beta}}{\hbar-\psi_{1}}\right|_{\mathcal{Z}_{\Gamma}} \frac{1}{\mathbf{e}\left(N \mathcal{Z}_{\Gamma}\right)} \\
& =\frac{C_{i}^{j}\left(d_{0}\right) u^{d_{0}}}{\hbar-\frac{\alpha_{j}-\alpha_{i}}{d_{0}}} \cdot\left(\left.\left\{u^{d_{c}} \int_{\mathcal{Z}_{\Gamma}} \frac{\mathbf{e}\left(\mathcal{V}_{0}^{\prime \prime}\right) \mathrm{ev}_{1}^{*} \phi_{j} \eta^{\beta}}{\hbar-\psi_{1}} \frac{1}{\mathbf{e}\left(N \mathcal{Z}_{\Gamma_{c}}\right)}\right\}\right|_{\hbar=\frac{\alpha_{j}-\alpha_{i}}{d_{0}}}\right) .
\end{aligned}
$$

We next sum (3.23) over $\Gamma \in A_{i}\left(j ; d_{0}\right)$. This is the same as summing the expression in the curly brackets over all $m$-pointed graphs with the marked point 1 attached to a vertex $v$ labeled $j$, i.e., all graphs of types $A_{j}$ and $B_{j}$. By the localization formula (3.9), the sum of the terms in the curly brackets 
over all such graphs $\Gamma_{c}$ is $\mathcal{Z}_{\eta, \beta}\left(\hbar, \alpha_{j}, u\right)$. Thus,

$$
\begin{gathered}
\left.\sum_{\Gamma \in A_{i}\left(j ; d_{0}\right)} u^{d_{0}+d_{c}} \int_{\mathcal{Z}_{\Gamma}} \frac{\mathbf{e}\left(\mathcal{V}_{0}^{\prime \prime}\right) \mathrm{ev}_{1}^{*} \phi_{i} \eta^{\beta}}{\hbar-\psi_{1}}\right|_{\mathcal{Z}_{\Gamma}} \frac{1}{\mathbf{e}\left(N \mathcal{Z}_{\Gamma}\right)} \\
=\frac{C_{i}^{j}\left(d_{0}\right) u^{d_{0}}}{\hbar-\frac{\alpha_{j}-\alpha_{i}}{d_{0}}} \cdot \mathcal{Z}_{\eta, \beta}\left(\left(\alpha_{j}-\alpha_{i}\right) / d_{0}, \alpha_{j}, u\right) .
\end{gathered}
$$

We conclude that $\mathcal{Z}_{\eta, \beta}(\hbar, x, u)$ is $C$-recursive in the sense of Definition 2.1:

- the middle term in (2.13) consists of the contributions from the graphs of type $B_{i}$;

- the $\left(d_{0}, j\right)$-summand in $(2.13)$ consists of the contributions from the graphs of type $A_{i}\left(j ; d_{0}\right)$.

\subsection{Proof of Lemma 1.2}

In this subsection we deduce Lemma 1.2 from Lemma 3.1, which is proved in the next subsection. The argument, in this subsection and the next one, is a modification on the proof of self-polynomiality of $\mathcal{Z}$ in Section 30.2 of [11].

We will denote the weight of the standard action of the one-torus $\mathbb{T}^{1}$ on $\mathbb{C}$ by $\hbar$. Thus, by Section 3.1,

$$
H_{\mathbb{T}^{1}}^{*} \approx \mathbb{Q}[\hbar], \quad H_{\widetilde{\mathbb{T}}}^{*} \approx \mathbb{Q}\left[\hbar, \alpha_{1}, \ldots, \alpha_{n}\right] \quad \Longrightarrow \quad \mathcal{H}_{\widetilde{\mathbb{T}}}^{*} \approx \mathbb{Q}_{\alpha}(\hbar)
$$

Throughout this subsection, $V=\mathbb{C} \oplus \mathbb{C}$ will denote the representation of $\mathbb{T}^{1}$ with the weights 0 and $-\hbar$. The induced action on $\mathbb{P} V$ has two fixed points:

$$
q_{1} \equiv[1,0], \quad q_{2} \equiv[0,1]
$$

Let $\gamma_{1} \longrightarrow \mathbb{P} V$ be the tautological line bundle. Then,

$$
\left.\mathbf{e}\left(\gamma_{1}^{*}\right)\right|_{q_{1}}=0,\left.\quad \mathbf{e}\left(\gamma_{1}^{*}\right)\right|_{q_{2}}=-\hbar, \quad \mathbf{e}\left(T_{q_{1}} \mathbb{P} V\right)=\hbar, \quad \mathbf{e}\left(T_{q_{2}} \mathbb{P} V\right)=-\hbar
$$

For each $d \in \overline{\mathbb{Z}}^{+}$, the action of $\widetilde{\mathbb{T}}$ on $\mathbb{C}^{n} \otimes \mathrm{Sym}^{d} V^{*}$ induces an action on

$$
\overline{\mathfrak{X}}_{d} \equiv \mathbb{P}\left(\mathbb{C}^{n} \otimes \operatorname{Sym}^{d} V^{*}\right)
$$

It has $(d+1) n$ fixed points:

$$
P_{i}(r) \equiv\left[\tilde{P}_{i} \otimes u^{d-r} v^{r}\right], \quad i \in[n], r \in\{0\} \cup[d],
$$


if $(u, v)$ are the standard coordinates on $V$ and $\tilde{P}_{i} \in \mathbb{C}^{n}$ is the $i$ th coordinate vector (so that $\left.\left[\tilde{P}_{i}\right]=P_{i} \in \mathbb{P}^{n-1}\right)$. Let

$$
\Omega \equiv \mathbf{e}\left(\gamma^{*}\right) \in H_{\widetilde{\mathbb{T}}}^{*}\left(\overline{\mathfrak{X}}_{d}\right)
$$

denote the equivariant hyperplane class.

For all $i \in[n]$ and $r \in\{0\} \cup[d]$,

$$
\left.\Omega\right|_{P_{i}(r)}=\alpha_{i}+r \hbar, \quad \mathbf{e}\left(T_{P_{i}(r)} \overline{\mathfrak{X}}_{d}\right)=\left.\left\{\prod_{\substack{s=0 \\ s, k) \neq(r, i)}}^{s=d} \prod_{\substack{k=1 \\ s=n}}^{\left.k=\alpha_{k}-r \hbar\right)}\right\}\right|_{\Omega=\alpha_{i}+r \hbar} \cdot{ }^{11}
$$

Since

$$
B \overline{\mathfrak{X}}_{d}=\mathbb{P}\left(B\left(\mathbb{C}^{n} \otimes \operatorname{Sym}^{d} V^{*}\right)\right) \longrightarrow B \widetilde{\mathbb{T}}
$$

and

$$
c\left(B\left(\mathbb{C}^{n} \otimes \operatorname{Sym}^{d} V^{*}\right)\right)=\prod_{s=0}^{s=d} \prod_{k=1}^{k=n}\left(1-\left(\alpha_{k}+s \hbar\right)\right) \in H^{*}(B \widetilde{\mathbb{T}}),{ }^{12}
$$

the $\widetilde{\mathbb{T}}$-equivariant cohomology of $\overline{\mathfrak{X}}_{d}$ is given by

$$
\begin{aligned}
H_{\tilde{\mathbb{T}}}^{*}\left(\overline{\mathfrak{X}}_{d}\right) & \equiv H^{*}\left(B \overline{\mathfrak{X}}_{d}\right)=H^{*}(B \widetilde{\mathbb{T}})[\Omega] / \prod_{s=0}^{s=d} \prod_{k=1}^{k=n}\left(\Omega-\left(\alpha_{k}+s \hbar\right)\right) \\
& \approx \mathbb{Q}\left[\Omega, \hbar, \alpha_{1}, \ldots, \alpha\right] / \prod_{s=0}^{s=d} \prod_{k=1}^{k=n}\left(\Omega-\alpha_{k}-s \hbar\right) \\
& \subset \mathbb{Q}_{\alpha}[\hbar, \Omega] / \prod_{s=0}^{s=d} \prod_{k=1}^{k=n}\left(\Omega-\alpha_{s}-r \hbar\right) .
\end{aligned}
$$

There is a natural $\widetilde{\mathbb{T}}$-equivariant morphism

$$
\Theta: \overline{\mathfrak{M}}_{0, m}\left(\mathbb{P} V \times \mathbb{P}^{n-1},(1, d)\right) \longrightarrow \overline{\mathfrak{X}}_{d}
$$

\footnotetext{
${ }^{11}$ The weight (i.e., negative first chern class) of the $\widetilde{\mathbb{T}}$-action on the line $P_{i}(r) \subset$ $\mathbb{C}^{n} \otimes \operatorname{Sym}^{d} V^{*}$ is $\alpha_{i}+r \hbar$. The tangent bundle of $\overline{\mathfrak{X}}_{d}$ at $P_{i}(r)$ is the direct sum of the lines $P_{i}(r)^{*} \otimes P_{k}(s)$ with $(k, s) \neq(i, r)$.

${ }^{12}$ The vector space $\mathbb{C}^{n} \otimes \operatorname{Sym}^{d} V^{*}$ is the direct sum of the one-dimensional representations $P_{k}(s)$ of $\widetilde{\mathbb{T}}$.
} 
A general element of $b$ of $\overline{\mathfrak{M}}_{0, m}\left(\mathbb{P} V \times \mathbb{P}^{n-1},(1, d)\right)$ determines a map

$$
(f, g): \mathbb{P}^{1} \longrightarrow\left(\mathbb{P} V, \mathbb{P}^{n}\right)
$$

up to an automorphism of the domain $\mathbb{P}^{1}$. Thus, the map

$$
g \circ f^{-1}: \mathbb{P} V \longrightarrow \mathbb{P}^{n-1}
$$

is well-defined and determines an element $\Theta(b) \in \overline{\mathfrak{X}}_{d}$. The map $\Theta$ extends continuously over the boundary of $\overline{\mathfrak{M}}_{0, m}\left(\mathbb{P} V \times \mathbb{P}^{n-1},(1, d)\right) .{ }^{13}$ We denote the restriction of $\Theta$ to the smooth substack

$\mathfrak{X}_{d} \equiv\left\{b \in \overline{\mathfrak{M}}_{0, m}\left(\mathbb{P} V \times \mathbb{P}^{n-1},(1, d)\right): \operatorname{ev}_{1}(b) \in q_{1} \times \mathbb{P}^{n-1}, \operatorname{ev}_{2}(b) \in q_{2} \times \mathbb{P}^{n-1}\right\}$

of $\overline{\mathfrak{M}}_{0, m}\left(\mathbb{P} V \times \mathbb{P}^{n-1},(1, d)\right)$ by $\theta_{d}$, or simply by $\theta$ whenever there is no ambiguity.

Let

$$
\pi: \overline{\mathfrak{M}}_{0, m}\left(\mathbb{P} V \times \mathbb{P}^{n-1},(1, d)\right) \longrightarrow \overline{\mathfrak{M}}_{0, m}\left(\mathbb{P}^{n-1}, d\right)
$$

be the natural projection map.

Lemma 3.1. With $\mathcal{Z}_{\eta, \beta}$ as in Lemma 1.2 and $\Phi$ as in (2.2),

$$
\begin{aligned}
(-\hbar)^{m-2} \Phi_{\mathcal{Z}, \mathcal{Z}_{\eta, \beta}}(\hbar, u, z)= & \sum_{d=0}^{\infty} u^{d} \int_{\mathfrak{X}_{d}} e^{\left(\theta^{*} \Omega\right) z} \pi^{*}\left(\mathbf{e}\left(\mathcal{V}_{0}^{\prime \prime}\right) \prod_{j=2}^{j=m}\left(\psi_{j}^{\beta_{j}} \mathrm{ev}_{j}^{*} \eta_{j}\right)\right) \\
& \times \prod_{j=3}^{j=m} \operatorname{ev}_{j}^{*}\left(\mathbf{e}\left(\gamma_{1}^{*}\right)\right) .
\end{aligned}
$$

Similarly Section 30.2 in [11], this lemma implies that

$$
(-\hbar)^{m-2} \Phi_{\mathcal{Z}, \mathcal{Z}_{\eta, \beta}}(\hbar, u, z) \in \mathbb{Q}_{\alpha}[\hbar][[u, z]]
$$

for the following reason. With $\eta^{\beta}$ as in (3.16), by (3.27)

$$
\theta_{d *}\left(\pi^{*}\left(\mathbf{e}\left(\mathcal{V}_{0}^{\prime \prime}\right) \eta^{\beta}\right) \prod_{j=3}^{j=m} \operatorname{ev}_{j}^{*}\left(\mathbf{e}\left(\gamma_{1}^{*}\right)\right)\right)=E_{\mathcal{Z}, \mathcal{Z}_{\eta, \beta} ; d}(\hbar, \Omega)
$$

\footnotetext{
${ }^{13}$ For a complete algebraic proof, see Lemma 2.6 in [11].
} 
for some $E_{\mathcal{Z}, \mathcal{Z}_{\beta, \eta} ; d} \in \mathbb{Q}_{\alpha}[\hbar, \Omega]$ of $\Omega$-degree at most $(d+1) n-1$. Therefore, by Lemma 3.1, (3.12), (3.9) and (3.26),

$$
\begin{aligned}
& (-\hbar)^{m-2} \Phi_{\mathcal{Z}, \mathcal{Z}_{\eta, \beta}}(\hbar, u, z) \\
& =\sum_{d=0}^{\infty} u^{d} \int_{\overline{\mathfrak{X}}_{d}} e^{\Omega z} \theta_{d *}\left(\pi^{*}\left(\mathbf{e}\left(\mathcal{V}_{0}^{\prime \prime}\right) \eta^{\beta}\right) \prod_{j=3}^{j=m} \mathrm{ev}_{j}^{*}\left(\mathbf{e}\left(\gamma_{1}^{*}\right)\right)\right) \\
& =\sum_{d=0}^{\infty} u^{d} \int_{\overline{\mathfrak{X}}_{d}} e^{\Omega z} E_{\mathcal{Z}, \mathcal{Z}_{\beta, \eta} ; d}(\hbar, \Omega)=\sum_{d=0}^{\infty} u^{d}\left(\sum_{r=0}^{r=d} \sum_{i=1}^{i=n} \frac{\left.\left.e^{\Omega z} E_{\mathcal{Z}, \mathcal{Z}_{\beta, \eta} ; d}(\hbar, \Omega)\right|_{P_{i}(r)}\right)}{\mathbf{e}\left(T_{P_{i}(r)} \overline{\mathfrak{X}}_{d}\right)}\right) \\
& =\sum_{d=0}^{\infty} u^{d}\left(\left.\sum_{r=0}^{r=d} \sum_{i=1}^{i=n} \frac{e^{\Omega z} E_{\mathcal{Z}, \mathcal{Z}_{\beta, \eta} ; d}(\hbar, \Omega)}{\prod_{s=0}^{s=d} \prod_{k=1}^{k=n}\left(\Omega-\alpha_{k}-s \hbar\right)}\right|_{\Omega=\alpha_{i}+r \hbar}\right) \\
& =\sum_{d=0}^{\infty} u^{d}\left(\frac{1}{2 \pi i k} \oint^{\Omega z} \frac{E_{\mathcal{Z}, \mathcal{Z}_{\beta, \eta} ; d}(\hbar, \Omega)}{\prod_{s=0}^{s=r} \prod_{k=1}^{k=n}\left(\Omega-\alpha_{k}-s \hbar\right)} d \Omega\right) .
\end{aligned}
$$

In the last expression, the integral has the same meaning as in Lemma 2.1. We have thus shown that $\mathcal{Z}_{\eta, \beta}$ is polynomial with respect to $\mathcal{Z}$, assuming Lemma 3.1.

\subsection{Proof of Lemma 3.1}

In this subsection we use the localization formula (3.9) to prove Lemma 3.1. We show that each fixed locus of the $\widetilde{\mathbb{T}}$-action on $\mathfrak{X}_{d}$ contributing to the righthand side of (3.29) corresponds to a pair $\left(\Gamma_{1}, \Gamma_{2}\right)$ of a graphs, with $\Gamma_{1}$ and $\Gamma_{2}$ contributing to $\mathcal{Z}\left(\hbar, \alpha_{i}, u e^{\hbar z}\right)$ and $(-\hbar)^{m-2} \mathcal{Z}_{\eta, \beta}\left(-\hbar, \alpha_{i}, u\right)$, respectively, for some $i \in[n]$.

Similarly to Section 3.2 , the fixed loci of the $\widetilde{\mathbb{T}}$-action on $\overline{\mathfrak{M}}_{0, m}(\mathbb{P} V \times$ $\left.\mathbb{P}^{n-1},\left(d^{\prime}, d\right)\right)$ correspond to decorated graphs $\Gamma$ with $m$ marked points and no loops. Each edge should be labeled by a pair of integers, indicating the degrees of the corresponding maps in $\mathbb{P} V$ and in $\mathbb{P}^{n-1}$. Each vertex should be labeled either $(1, j)$ or $(2, j)$ for some $j \in[n]$, indicating the fixed point, $\left(q_{1}, P_{j}\right)$ or $\left(q_{2}, P_{j}\right)$, to which the vertex is mapped. No two consecutive vertex labels are the same, but if two consecutive vertex labels differ in the $k$ th component, with $k=1,2$, the $k$ th component of the label for edge connecting them must be nonzero.

The situation for the $\widetilde{\mathbb{T}}$-action on

$$
\mathfrak{X}_{d} \subset \overline{\mathfrak{M}}_{0, m}\left(\mathbb{P} V \times \mathbb{P}^{n-1},(1, d)\right)
$$


is simpler, however. There is a unique edge of positive $\mathbb{P} V$-degree. We draw it as a thick horizontal line. The first component of all other edge labels must be 0 ; so we drop it. The first components of the vertex labels change only when the thick edge is crossed. Thus, we drop the first components of the vertex labels as well, with the convention that these components are 1 on the left side of the thick edge and 2 on the right. In particular, the vertices to the left of the thick edge (including the left endpoint) lie in $q_{1} \times \mathbb{P}^{n-1}$ and the vertices to its right lie in $q_{2} \times \mathbb{P}^{n-1}$. Thus, by (3.28), the marked point 1 is attached to a vertex to the left of the thick edge and the marked point 2 is attached to a vertex to the right. Furthermore, by the first identity in (3.25), such a graph will not contribute to the right-hand side of (3.29) unless the remaining marked points are also attached to vertices to the right of the thick edge. Finally, both vertices of the thick edge have the same (remaining, second) label $i \in[n]$. Let $\mathcal{A}_{i}$ denote the set of graphs as above so that the two endpoints of the thick edge are labeled $i$; see Figure 3.

If $\Gamma \in \mathcal{A}_{i}$, we break it into three sub-graphs:

(i) $\Gamma_{1}$ consisting of all vertices and edges of $\Gamma$ to the left of the thick edge, including its left vertex $v_{1}$, and a new marked point 2 attached to $v_{1}$;

(ii) $\Gamma_{0}$ consisting of the thick edge, its two vertices $v_{1}$ and $v_{2}$ and new marked points 1 and 2 attached to $v_{1}$ and $v_{2}$, respectively;

(iii) $\Gamma_{2}$ consisting of all vertices and edges of $\Gamma$ to the right of the thick edge, including its right vertex $v_{2}$, and a new marked point 1 attached to $v_{2}$

see Figure 4. The fixed locus in $\mathfrak{X}_{d}$ corresponding to $\Gamma$ is then

$$
\mathcal{Z}_{\Gamma} \approx \mathcal{Z}_{\Gamma_{1}} \times \mathcal{Z}_{\Gamma_{0}} \times \mathcal{Z}_{\Gamma_{2}}
$$

The middle term is a single point. Let $\pi_{1}, \pi_{0}$ and $\pi_{2}$ denote the three component projection maps. Denote by $d_{1}$ and $d_{2}$ be the degrees of $\Gamma_{1}$ and

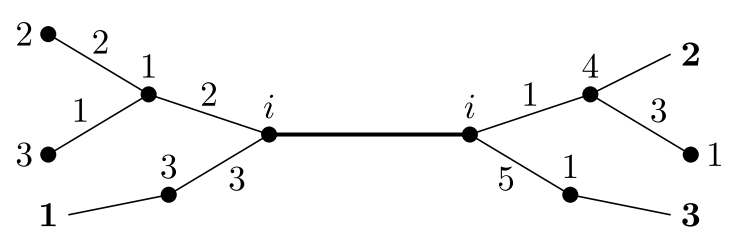

Figure 3: A graph representing a fixed locus in $\mathfrak{X}_{d} ; i \neq 1,3,4$. 

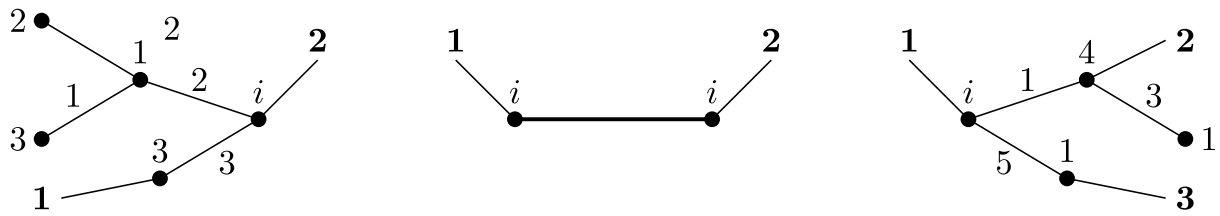

Figure 4: The three sub-graphs of the graph in Figure 3.

$\Gamma_{2}$, i.e.,

$$
\mathcal{Z}_{\Gamma_{1}} \subset \overline{\mathfrak{M}}_{0,2}\left(\mathbb{P}^{n-1}, d_{1}\right), \quad \mathcal{Z}_{\Gamma_{2}} \subset \overline{\mathfrak{M}}_{0, m}\left(\mathbb{P}^{n-1}, d_{2}\right)
$$

The exceptional case for the first statement is $d_{1}=0$, in which case the corresponding moduli space does not exist.

Suppose $\Gamma \in \mathcal{A}_{i}, d_{1}$ and $d_{2}$ are as above, and $d_{1}>0$. Similarly to (3.19),

$$
\begin{aligned}
\left.\pi^{*} \mathcal{V}_{0}^{\prime \prime}\right|_{\mathcal{Z}_{\Gamma}} & =\pi_{1}^{*} \mathcal{V}_{0}^{\prime \prime} \oplus \pi_{2}^{*} \mathcal{V}_{0}^{\prime \prime} \\
\frac{\mathcal{N} \mathcal{Z}_{\Gamma}}{T_{P_{i}} \mathbb{P}^{n-1}} & =\pi_{1}^{*}\left(\frac{\mathcal{N} \mathcal{Z}_{\Gamma_{1}}}{T_{P_{i}} \mathbb{P}^{n-1}}\right) \oplus \pi_{2}^{*}\left(\frac{\mathcal{N}_{\Gamma_{2}}}{T_{P_{i}} \mathbb{P}^{n-1}}\right) \oplus \pi_{1}^{*} L_{2} \otimes \pi_{0}^{*} L_{1} \oplus \pi_{0}^{*} L_{2} \otimes \pi_{2}^{*} L_{1}
\end{aligned}
$$

where $\mathcal{N} \mathcal{Z}_{\Gamma} \longrightarrow \mathcal{Z}_{\Gamma}$ is the normal bundle of $\mathcal{Z}_{\Gamma}$ in $\mathfrak{X}_{d}$ and $L_{2} \longrightarrow \mathcal{Z}_{\Gamma_{1}}$, $L_{1}, L_{2} \longrightarrow \mathcal{Z}_{\Gamma_{0}}$ and $L_{1} \longrightarrow \mathcal{Z}_{\Gamma_{2}}$ are the tautological tangent line bundles. We note that

$$
L_{1}=T_{q_{1}} \mathbb{P}^{1} \quad \text { and } \quad L_{2}=T_{q_{2}} \mathbb{P}^{1} \quad \text { on } \quad \mathcal{Z}_{\Gamma_{0}}
$$

Thus, by (3.31), (3.8) and (3.25),

$$
\begin{aligned}
\left.\pi^{*}\left(\mathbf{e}\left(\mathcal{V}_{0}^{\prime \prime}\right) \eta^{\beta}\right) \prod_{j=3}^{j=m} \mathrm{ev}_{j}^{*}\left(\mathbf{e}\left(\gamma^{*}\right)\right)\right|_{\mathcal{Z}_{\Gamma}}= & \pi_{1}^{*} \mathbf{e}\left(\mathcal{V}_{0}^{\prime \prime}\right) \cdot \pi_{2}^{*}\left(\mathbf{e}\left(\mathcal{V}_{0}^{\prime \prime}\right) \eta^{\beta}(-\hbar)^{m-2}\right) \\
\frac{\prod_{k \neq i}\left(\alpha_{i}-\alpha_{k}\right)}{\mathbf{e}\left(N \mathcal{Z}_{\Gamma}\right)}= & \pi_{1}^{*}\left(\frac{\mathrm{ev}_{2}^{*} \phi_{i}}{\mathbf{e}\left(\mathcal{N} \mathcal{Z}_{\Gamma_{0}}\right)}\right) \cdot \pi_{2}^{*}\left(\frac{\mathrm{ev}_{1}^{*} \phi_{i}}{\mathbf{e}\left(\mathcal{N} \mathcal{Z}_{\Gamma_{0}}\right)}\right) \\
& \cdot \frac{1}{\hbar-\pi_{1}^{*} \psi_{2}} \cdot \frac{1}{(-\hbar)-\pi_{2}^{*} \psi_{1}}
\end{aligned}
$$

The map $\theta$ takes the locus $\mathcal{Z}_{\Gamma}$ to a fixed point $P_{k}(r) \in \overline{\mathfrak{X}}_{d}$. It is immediate that $k=i$. By continuity considerations, $r=d_{1}$. Thus, by the first identity 
in $(3.26)$,

$$
\left.\theta^{*} \Omega\right|_{\mathcal{Z}_{\Gamma}}=\alpha_{i}+d_{1} \hbar
$$

Combining (3.30) and (3.32) with this observation, we obtain

$$
\begin{aligned}
& \int_{\mathcal{Z}_{\Gamma}} \frac{\left.e^{\left(\theta^{*} \Omega\right) z} \pi^{*}\left(\mathbf{e}\left(\mathcal{V}_{0}^{\prime \prime}\right) \eta^{\beta}\right) \prod_{j=3}^{j=m} \mathrm{ev}_{j}^{*}\left(\mathbf{e}\left(\gamma^{*}\right)\right)\right|_{\mathcal{Z}_{\Gamma}}}{\mathbf{e}\left(\mathcal{N} \mathcal{Z}_{\Gamma}\right)} \\
& \quad=(-\hbar)^{m-2} \frac{e^{\alpha_{i} z}}{\prod_{k \neq i}\left(\alpha_{i}-\alpha_{k}\right)}\left\{\left.e^{d_{1} \hbar z} \int_{\mathcal{Z}_{\Gamma_{1}}} \frac{\mathbf{e}\left(\mathcal{V}_{0}^{\prime \prime}\right) \mathrm{ev}_{2}^{*} \phi_{i}}{\hbar-\psi_{2}}\right|_{\mathcal{Z}_{\Gamma_{1}}} \frac{1}{\mathbf{e}\left(\mathcal{N} \mathcal{Z}_{\Gamma_{1}}\right)}\right\} \\
& \quad \times\left\{\left.\int_{\mathcal{Z}_{\Gamma_{2}}} \frac{\mathbf{e}\left(\mathcal{V}_{0}^{\prime \prime}\right) \eta^{\beta}}{(-\hbar)-\psi_{1}}\right|_{\mathcal{Z}_{\Gamma_{2}}} \frac{1}{\mathbf{e}\left(\mathcal{N} \mathcal{Z}_{\Gamma_{2}}\right)}\right\}
\end{aligned}
$$

We note that this identity remains valid for $d_{1}=0$ if we set the term in the first curly brackets to 1 for $d_{1}=0$.

We now sum up (3.33), multiplied by $u^{d_{1}+d_{2}}$, over all $\Gamma \in \mathcal{A}_{i}$. This is the same as summing over all pairs $\left(\Gamma_{1}, \Gamma_{2}\right)$ of graphs such that

(1) $\Gamma_{1}$ is a 2-pointed graph of a degree $d_{1} \geq 0$ such that the marked point 2 is attached to the vertex labeled $i$;

(2) $\Gamma_{2}$ is an $m$-pointed graph of a degree $d_{2} \geq 0$ such that the marked point 1 is attached to the vertex labeled $i$.

By the localization formula (3.9),

$$
\begin{gathered}
\sum_{\Gamma_{1}} u^{d_{1}}\left\{\left.e^{d_{1} \hbar z} \int_{\mathcal{Z}_{\Gamma_{1}}} \frac{\mathbf{e}\left(\mathcal{V}_{0}^{\prime \prime}\right) \mathrm{ev}_{2}^{*} \phi_{i}}{\hbar-\psi_{2}}\right|_{\mathcal{Z}_{\Gamma_{1}}} \frac{1}{\mathbf{e}\left(\mathcal{N} \mathcal{Z}_{\Gamma_{1}}\right)}\right\} \\
=1+\sum_{d=1}^{\infty}\left(u e^{\hbar z}\right)^{d} \int_{\overline{\mathfrak{M}}_{0,2}\left(\mathbb{P}^{n-1}, d\right)} \frac{\mathbf{e}\left(\mathcal{V}_{0}^{\prime \prime}\right)}{\hbar-\psi_{2}} \mathrm{ev}_{2}^{*} \phi_{i} \\
=1+\sum_{d=1}^{\infty}\left(u e^{\hbar z}\right)^{d} \int_{\overline{\mathfrak{M}}_{0,2}\left(\mathbb{P}^{n-1}, d\right)} \frac{\mathbf{e}\left(\mathcal{V}_{0}^{\prime}\right)}{\hbar-\psi_{1}} \mathrm{ev}_{1}^{*} \phi_{i} \\
=\mathcal{Z}\left(\hbar, \alpha_{i}, u e^{\hbar z}\right), \\
\sum_{\Gamma_{2}} u^{d_{2}}\left\{\left.\int_{\mathcal{Z}_{\Gamma_{2}}} \frac{\mathbf{e}\left(\mathcal{V}_{0}^{\prime \prime}\right) \eta^{\beta} \mathrm{ev}_{1}^{*} \phi_{i}}{(-\hbar)-\psi_{1}}\right|_{\mathcal{Z}_{\Gamma_{2}}} \frac{1}{\mathbf{e}\left(\mathcal{N}_{\left.\mathcal{Z}_{\Gamma_{2}}\right)}\right.}\right\}
\end{gathered}
$$




$$
\begin{aligned}
& =\sum_{d=0}^{\infty} u^{d} \int_{\overline{\mathfrak{M}}_{0, m}\left(\mathbb{P}^{n-1}, d\right)} \frac{\mathbf{e}\left(\mathcal{V}_{0}^{\prime \prime}\right) \eta^{\beta} \mathrm{ev}_{1}^{*} \phi_{i}}{(-\hbar)-\psi_{1}} \\
& =\mathcal{Z}_{\eta, \beta}\left(-\hbar, \alpha_{i}, u\right) .
\end{aligned}
$$

Finally, by (3.9), (3.33) and (3.34),

$$
\begin{aligned}
& \int_{\mathfrak{X}_{d}} e^{\left(\theta^{*} \Omega\right) z} \pi^{*}\left(\mathbf{e}\left(\mathcal{V}_{0}^{\prime \prime}\right) \eta^{\beta}\right) \prod_{j=3}^{j=m} \operatorname{ev}_{j}^{*}\left(\mathbf{e}\left(\gamma_{1}^{*}\right)\right) \\
& =(-\hbar)^{m-2} \sum_{i=1}^{i=n} \frac{e^{\alpha_{i} z}}{\prod_{k \neq i}\left(\alpha_{i}-\alpha_{k}\right)} \mathcal{Z}\left(\hbar, \alpha_{i}, u e^{\hbar z}\right) \mathcal{Z}_{\eta, \beta}\left(-\hbar, \alpha_{i}, u\right) \\
& =(-\hbar)^{m-2} \Phi_{\mathcal{Z}, \mathcal{Z}_{\eta, \beta}}(\hbar, u, z)
\end{aligned}
$$

as claimed in (3.29).

\section{Acknowledgment}

Partially supported by a Sloan fellowship and DMS Grant 0604874.

\section{References}

[1] M. Atiyah and R. Bott, The moment map and equivariant cohomology, Topology 23 (1984), 1-28.

[2] M. Bershadsky, S. Cecotti, H. Ooguri and C. Vafa, Holomorphic anomalies in topological field theories, Nucl. Phys. B405 (1993), 279-304.

[3] A. Bertram, Another way to enumerate rational curves with torus actions, Invent. Math. 142(3) (2000), 487-512.

[4] P. Candelas, X. de la Ossa, P. Green and L. Parkes, A pair of calabi-yau manifolds as an exactly soluble superconformal theory, Nuclear Phys. B359 (1991), 21-74.

[5] A. Gathmann, Absolute and relative Gromov-Witten invariants of very ample hypersurfaces, Duke Math. J. 115(2) (2002), 171-203.

[6] A. Givental, The mirror formula for quintic threefolds, Amer. Math. Soc. Transl. Ser. 2, 196 (1999), 49-62.

[7] A. Klemm and R. Pandharipande, Enumerative geometry of CalabiYau 4-folds, Comm. Math. Phys. 281(3) (2008), 621-653. 
[8] Y. P. Lee, Quantum Lefschetz hyperplane theorem, Invent. Math. 145(1) (2001), 121-149.

[9] Y. P. Lee and R. Pandharipande, A reconstruction theorem in quantum cohomology and quantum K-theory, Amer. J. Math. 126(6) (2004), 1367-1379.

[10] B. Lian, K. Liu and S.T. Yau, Mirror principle I, Asian J. Math. 1(4) (1997), 729-763.

[11] K. Hori, S. Katz, A. Klemm, R. Pandharipande, R. Thomas, C. Vafa, R. Vakil and E. Zaslow, Mirror symmetry, Clay Math. Inst., Amer. Math. Soc., 2003.

[12] R. Pandharipande, Intersections of $\mathbb{Q}$-divisors on Kontsevich's moduli space $\bar{M}_{0, n}\left(\mathbb{P}^{r}, d\right)$ and Enumerative Geometry, Trans. Amer. Math. Soc. 351(4) (1999), 1481-1505.

[13] D. Zagier and A. Zinger, Some properties of hypergeometric series associated with mirror symmetry, Modular Forms and String Duality, Fields Inst. Commun. 54, AMS, Providence, RI, 2008, 163-177.

[14] A. Zinger, The reduced genus-one Gromov-Witten invariants of CalabiYau hypersurfaces, J. Amer. Math. Soc. 22(3) (2009), 691-737.

Department of Mathematics

SUNY STONY BROOK

NY 11794-3651, USA

E-mail address: azinger@math.sunysb.edu

ReCeived Month 00, 2010 
